\begin{tabular}{|c|c|}
\hline Title & $\begin{array}{l}\text { Numerical testing of quantitative phase field models with different polynomials for isothermal solidification in binary } \\
\text { alloys }\end{array}$ \\
\hline Author(s) & Ohno, Munekazu; Takaki, Tomohiro; Shibuta, Y asushi \\
\hline Citation & $\begin{array}{l}\text { Journal of computational physics, 335, 621-636 } \\
\text { https://doi.org/10.1016/.jcp.2017.01.053 }\end{array}$ \\
\hline Issue Date & $2017-0415$ \\
\hline Doc URL & http:/hdl. handle.net/2115/73577 \\
\hline Rights & $\begin{array}{l}\text { (9 2017. This manuscript version is made available under the CC-BY -NC-ND } 4.0 \text { license } \\
\text { http://reativecommons.org/icenses/by-nc-nd/4.0/ }\end{array}$ \\
\hline Rights(URL) & http://creativecommons.org/icenses/by-nc-nd/4.0/ \\
\hline Type & article (author version) \\
\hline File Information & 20170hno_JCP_HUSCAP.pdf \\
\hline
\end{tabular}

Instructions for use 


\title{
Numerical testing of quantitative phase-field models with different polynomials for isothermal solidification in binary alloys
}

\author{
Munekazu Ohno ${ }^{1}$, Tomohiro Takaki ${ }^{2}$ and Yasushi Shibuta ${ }^{3}$ \\ ${ }^{1}$ Division of Materials Science and Engineering, Faculty of Engineering, Hokkaido University, \\ Kita 13 Nishi 8, Kita-ku, Sapporo, Hokkaido 060-8628, Japan \\ ${ }^{2}$ Faculty of Mechanical Engineering, Kyoto Institute of Technology, \\ Matsugasaki, Sakyo-ku, Kyoto 606-8585, Japan \\ ${ }^{3}$ Department of Materials Engineering, The University of Tokyo, \\ 7-3-1 Hongo, Bunkyo-ku, Tokyo 113-8656, Japan
}

\begin{abstract}
Quantitative phase-field models have been developed as feasible computational tools for solving the free-boundary problem in solidification processes. These models are constructed with some polynomials of the phase-field variable that describe variations of the physical quantities inside the diffuse interface. The accuracy of the simulation depends on the choice of the polynomials and such dependence is indispensable for high-performance computing and valuable for extending the range of applications of the model to several physical systems. However, little is known about the dependence of the accuracy on the choice of the polynomials. In this study, numerical testing is carried out for quantitative phase-field models with extensive sets of polynomials (24 different models) for isothermal solidification in binary alloys. It is demonstrated in two-dimensional simulations of dendritic growth that a specific set of polynomials must be employed to achieve high accuracy in the models with double-well and double-obstacle potentials. Both types of model with the best set of polynomials yield almost the same numerical accuracy.
\end{abstract}

Keywords: Phase-field simulation; Convergence test; Solidification; Alloy, Dendrite; 


\section{Introduction}

Accurate control of the solidification microstructures in alloys is an issue of great importance in the field of metallurgy because microstructural features such as the size and morphology of the solidified grains and microsegregation directly determine the properties of the as-cast alloys. Several methods have been developed for simulation of solidification microstructures on the basis of the sharp-interface description [1-4] and also the diffuse-interface description. The phase-field model is a diffuse-interface model for describing microstructural processes in solidification [5-9]. It serves as a viable computational tool for solving the free-boundary problem (FBP) of solid-liquid interfaces. Although phase-field model generally has a high computational cost, recent advances in parallel computing techniques have enabled large-scale phase-field simulations of the competitive growth of a bunch of dendrites $[9,10]$. Furthermore, progress has been made in evaluating the input parameters in the phase-field model on the basis of atomistic simulations [10-12]. Such progress has rapidly increased the effectiveness of phase-field simulations for analyzing and controlling solidification microstructures.

In the phase-field model, the interface is not sharp but a diffuse entity having a thickness. Since state variables such as the order parameter called the phase-field variable and the concentration field(s) change continuously inside the interface, the computational cost increases with decreasing interface thickness. The interface thickness must be chosen to be much larger than the atomic distance but smaller than any length scale relevant to the microstructure (e.g., the tip radius of a dendrite). Therefore, the interface thickness in this model is a constant determined by the scale of microstructure and the computational cost. When a finite value is assigned to the thickness, however, it is difficult to use early models in a quantitative manner. The early models are called standard phase-field models in this paper. This problem arises because the standard models were constructed to reproduce the solution of the FBP in the limit of zero thickness (the sharp-interface limit). In an actual simulation with a finite thickness, they suffer from unphysical magnification of the interface effects, causing an undesired dependence of the simulation result on the thickness. This serious problem was resolved in so-called quantitative phase-field models [13-21]. These quantitative models were constructed on the basis of the thin-interface asymptotics, where the model is mapped onto the FBP in the limit of non-zero thickness. The first quantitative model was proposed for solidification in a pure substance with symmetric diffusion (i.e., equal thermal diffusivity in the liquid and solid phases) [13], and it was extended to deal with alloy solidification with one-sided diffusion [13-18] (negligible solute diffusivity in the solid) and also two-sided asymmetric diffusion (non-zero solute diffusivity in the solid) [19-21]. The essential ingredient in the quantitative phase-field models for alloy systems is the so-called anti-trapping current, a correction term for the diffusion flux inside the interface [14]. Although this correction term was introduced in a phenomenological manner in the early models, variational formulations of quantitative phase-field 
models including the anti-trapping current have recently been demonstrated for a pure substance [22] and for a binary alloy system with two-sided asymmetric diffusion [23].

Quantitative phase-field models were developed as an effective alternative to the FBP and have been increasingly utilized to simulate solidification microstructures [24-32]. It is important to point out that there are several possible forms of quantitative phase-field models and that not all the models yield accurate numerical solutions. In the phase-field models, continuous variations of the physical quantities inside the interface are described by polynomials of the phase-field variable, which are called interpolating functions in this paper. As demonstrated in an early study [13], different forms of the interpolating function related to the enthalpy result in different numerical accuracy for dendritic growth in a pure substance. In the case of isothermal solidification in a binary alloy, which is our main concern, four types of interpolating function must be introduced to represent the continuous variations of the barrier potential between the solid and liquid, the bulk's free energy densities (driving force), the concentration field and the diffusivities. There are various possible forms for each interpolating function and a different set of functions should result in different numerical accuracy. However, little has been clarified regarding the accuracy of models with different sets of interpolating functions. For instance, either the double-well potential [13-29] or the double-obstacle potential [30-36] has been employed to represent the barrier potential in the standard and quantitative phase-field models. Each potential offers different advantages in terms of ease of implementation, applicability to multiphase systems and so forth. However, it is not clear which potential is superior in terms of numerical accuracy because a fair comparison between them has not been carried out in the framework of quantitative simulations. Note that only a few sets of interpolating functions have so far been employed in quantitative phase-field simulations [13-32]. Models with the other sets of functions have not yet been implemented and hence they have not been subjected to numerical testing. The dependence of the numerical accuracy on the choice of interpolating functions is important information in the development of quantitative phase-field models for a variety of physical systems. In addition, finding the best set of interpolating functions is an issue of great interest in the high-performance computing of solidification microstructures.

The main purpose of this study is to elucidate the dependence of the numerical accuracy of quantitative phase-field models on the choice of the interpolating functions and also to find the best set of functions by performing detailed comparisons of the numerical accuracy between them. The numerical testing is carried out for 24 different quantitative models. It is found that models constructed with the double-well and double-obstacle potentials yield comparably good numerical accuracy as long as the best set of interpolating functions is employed. In the next section, we provide a brief explanation of the quantitative phase-field models and interpolating functions. Detailed comparisons of the accuracy between the models are demonstrated by performing one-dimensional simulations of a moving flat interface and two-dimensional simulations of dendritic 
growth in Sec. 3. The conclusions are given in Sec. 4.

\section{Quantitative phase-field models and computational details}

\subsection{Model for isothermal solidification in a dilute binary alloy}

In this study, we focus on isothermal solidification in a dilute binary alloy which is a simple case suitable for the present purpose. We consider a dilute alloy with a constant partition coefficient $k$, constant diffusivity in the liquid $D_{l}$ and negligible solid diffusivity (i.e., one-sided diffusion). Then, the sharp-interface equations for the moving solid-liquid interface are given as

$$
\begin{aligned}
& \partial_{t} c_{l}=D_{l} \nabla^{2} c_{l}, \\
& (1-k) c_{l} V_{n}=-\left.D_{l} \partial_{\eta} c_{l}\right|^{*}, \\
& \frac{c_{l}^{*}}{c_{l}^{e}}=1-(1-k) d_{0} K-(1-k) \beta V_{n},
\end{aligned}
$$

where $c_{l}$ is the concentration in the liquid phase, $\left.\partial_{\eta} c_{l}\right|^{*}$ is the concentration gradient in the normal direction to the solid-liquid interface on the liquid side of the interface, $c_{l}{ }^{*}$ is the liquid concentration at the interface, $c_{l}{ }^{e}$ is the equilibrium concentration in the liquid at the holding temperature, $d_{0}$ is the chemical capillary length, $K$ is the mean curvature, $\beta$ is the inverse of the linear kinetic coefficient and $V_{n}$ is the normal velocity of the solid-liquid interface. In Eq. (3), anisotropic effects are omitted for simplicity.

In the quantitative phase-field models, the microstructural evolution process is described by the spatial and temporal variations of the phase-field variable $\phi$ and concentration field $c$. $\phi$ takes values of +1 in the solid and -1 in the liquid and it continuously changes from +1 to -1 inside the solid-liquid interface. For expedience, we use the dimensionless concentration field $u$ defined as $u=$ $\left(c_{l}-c_{l}{ }^{e}\right) /\left[(1-k) c_{l}{ }^{e}\right]$ instead of the concentration field $c$. The time evolution equations of $\phi$ and $u$ in the two-dimensional system are written as $[15,19]$,

$$
\begin{array}{rr}
\tau a_{s}(\vec{n})^{2} \partial_{t} \phi=W^{2} \nabla \cdot\left[a_{s}(\vec{n})^{2} \nabla \phi\right]+W^{2} \sum_{i=x, y} \partial_{i}\left[|\nabla \phi|^{2} a_{s}(\vec{n}) \frac{\partial a_{s}(\vec{n})}{\partial\left(\partial_{i} \phi\right)}\right] & -\frac{d f(\phi)}{d \phi}-\frac{d g(\phi)}{d \phi} a_{1} \frac{W}{d_{0}} u \\
\frac{1}{2}(1+k-(1-k) h(\phi)) \partial_{t} u &
\end{array}
$$




$$
=\nabla\left[D_{l} q(\phi) \nabla u+\vec{J}_{A T}\right]+\frac{1}{2}(1+(1-k) u) \partial_{t} h(\phi),
$$

with

$$
\begin{aligned}
& \tau=\frac{\beta W^{2}}{d_{0}}+\frac{a_{1} a_{2} W^{3}(1+(1-k) u)}{D_{l} d_{0}}, \\
& \vec{n}=-\nabla \phi /|\nabla \phi|, \\
& a_{s}(\vec{n})=\left(1-3 \varepsilon_{4}\right)\left[1+\frac{4 \varepsilon_{4}}{1-3 \varepsilon_{4}} \frac{\left(\partial_{\chi} \phi\right)^{4}+\left(\partial_{y} \phi\right)^{4}}{|\nabla \phi|^{4}}\right], \\
& \vec{J}_{A T}=a_{A T}(\phi)(1+(1-k) u) W \partial_{t} \phi \frac{\nabla \phi}{|\nabla \phi|} .
\end{aligned}
$$

Here $\tau$ is the relaxation constant for $\phi$, $W$ is a measure of the interface thickness as explained later and $\vec{n}$ is the unit vector normal to the interface. We included the crystalline anisotropy in Eq. (4), where $a_{s}(\vec{n})$ given by Eq. (8) is the function describing a fourfold crystalline anisotropy and $\varepsilon_{4}$ represents the strength of anisotropy of the interfacial energy. $\vec{J}_{A T}$ given by Eq. (9) is the anti-trapping current, which corrects the diffusion flux inside the interface, making the model consistent with the sharp-interface equations (1)-(3). $a_{A T}(\phi)$ is given as [15]

$$
a_{A T}(\phi)=\frac{(h(\phi)-1)(1-q(\phi))}{2 \partial \phi / \partial \eta^{\prime}},
$$

where $\eta^{\prime}=\eta / W$ and $\eta$ is the spatial coordinate in the direction normal to the interface and

$$
\partial \phi / \partial \eta^{\prime}=-\sqrt{2 f(\phi)} .
$$

In Eqs. (4), (5), (10) and (11), there are four interpolating functions, $f(\phi), g(\phi), h(\phi)$ and $q(\phi) . f(\phi)$ represents the barrier potential between the solid and liquid phases, exhibiting the minima at $\phi= \pm 1$. $g(\phi)$ is an interpolating function between the bulk's free energy densities of the solid and liquid and is usually a monotonically increasing function of $\phi . h(\phi)$ and $q(\phi)$ are interpolating functions for the concentration and diffusivity, respectively, with $h( \pm 1)= \pm 1, q(+1)=0$ and $q(-1)=1$. To achieve a correct mapping of the model onto the sharp-interface equations (1)-(3), these functions must satisfy the following constraints;

$$
\int_{0}^{+1}(h(\phi)-h(+1)) \frac{d \phi}{\partial \phi / \partial \eta^{\prime}}=\int_{0}^{-1}(h(\phi)-h(-1)) \frac{d \phi}{\partial \phi / \partial \eta^{\prime}}=H,
$$




$$
\int_{0}^{+1}(q(\phi)-q(+1)) \frac{d \phi}{\partial \phi / \partial \eta^{\prime}}=\int_{0}^{-1}(q(\phi)-q(-1)) \frac{d \phi}{\partial \phi / \partial \eta^{\prime}}=Q .
$$

For simplicity, $q(\phi)$ is defined in this study as

$$
q(\phi)=\frac{1-h(\phi)}{2} .
$$

Then, the constraint of Eq. (13) becomes equivalent to Eq. (12). Equation (14) has been commonly employed in previous works $[15,19]$. Furthermore, the constants $a_{1}$ and $a_{2}$ in Eqs. (4) and (6) are given as

$$
\begin{aligned}
& a_{1}=\frac{I}{J}, \\
& a_{2}=\frac{J H+K}{2 I},
\end{aligned}
$$

where

$$
\begin{aligned}
& I=\sqrt{2} \int_{-1}^{+1} \sqrt{f(\phi)} d \phi, \\
& J=g(+1)-g(-1), \\
& K=\int_{0}^{-1}(g(-1)-g(\phi)) h(\phi) \frac{d \phi}{\partial \phi / \partial \eta^{\prime}}-\int_{0}^{+1}(g(+1)-g(\phi)) h(\phi) \frac{d \phi}{\partial \phi / \partial \eta^{\prime}} .
\end{aligned}
$$

Further detail on the quantitative phase-field models can be found in Refs. [15, 19].

Several forms for $f(\phi), g(\phi)$ and $h(\phi)$ have been employed in the standard and quantitative phase-field models [13-35]. As mentioned in the introduction, the numerical accuracy of the quantitative phase-field model (and standard models) depends on the chosen forms of the interpolating functions. In this paper, we investigate the numerical accuracy of the models with different sets of interpolating functions.

\subsection{Interpolating functions}

The interpolating functions tested in this study are explained in this section. Hereafter, $f(\phi)$, $g(\phi)$ and $h(\phi)$ are respectively denoted as $f_{p}(\phi), g_{q}(\phi)$ and $h_{r}(\phi)$, where the subscripts $p, q$ and $r$ specify different forms of the functions.

The interpolating function $f_{p}(\phi)$ should be of primary importance in the model because it determines the steady-state profile of $\phi$ across the interface (traveling wave solution). Two types of function, specifically the double-well potential $f_{1}(\phi)$ and double-obstacle potential $f_{2}(\phi)$, have been employed in previous models [13-35]. These are given as

$$
f_{1}(\phi)=\frac{1}{4}\left(1-\phi^{2}\right)^{2}
$$




$$
f_{2}(\phi)=\frac{1}{2}\left(1-\phi^{2}\right) .
$$

In the latter case, the obstacle potentials are introduced at $\phi= \pm 1$, viz., $f_{2}(\phi)=\infty$ for $|\phi| \geq 1$. The first derivatives of these functions are listed in Table 1. From Eq. (4), in two-phase equilibrium $\left(\partial_{t} \phi\right.$ $=0$ and $u=0$ ), the following spatial profile of $\phi$ across the interface is obtained for $f_{1}(\phi)$;

$$
\phi=-\tanh \left(\frac{\eta}{\sqrt{2} W_{D W}}\right),
$$

where the boundary conditions $\phi \rightarrow-1$ for $\eta \rightarrow+\infty$ and $\phi \rightarrow+1$ for $\eta \rightarrow-\infty$ are considered and the anisotropy is neglected. $W_{D W}$ is identical to $W$ in Eq. (4). Under the same boundary conditions, the spatial profile of $\phi$ for $f_{2}(\phi)$ is given as

$$
\phi=-\sin \left(\frac{\eta}{W_{D O}}\right),
$$

for $-(\pi / 2) W_{D O} \leq \eta \leq(\pi / 2) W_{D O}, \phi=-1$ for $\eta>(\pi / 2) W_{D O}$ and $\phi=+1$ for $\eta<-(\pi / 2) W_{D O}$. Here, $W_{D O}$ corresponds to $W$ in Eq. (4).

The double-well potential has been employed in most quantitative phase-field simulations [13-29]. In this potential, $\mathrm{d} f_{1} / \mathrm{d} \phi=0$ is satisfied at $\phi= \pm 1$ and no special care is required for the calculation of $\partial_{t} \phi$ (Eq. (4)) near $\phi= \pm 1$. In the case of the double-obstacle potential, however, $\mathrm{d} f_{2} / \mathrm{d} \phi$ $\neq 0$ at $\phi= \pm 1$ and hence the range of $|\phi|>1$ should be manually prohibited in the numerical integration of Eq. (4). The double-obstacle potential has the advantage that the interfacial region is well defined as the region of $|\phi|<1$ with thickness $\pi W_{D O}$. Moreover, this potential is beneficial for modeling multiphase systems [33-36]. Quantitative phase-field simulations with this potential were reported in Refs. [30, 32]. One of these functions has been adopted in many works in the light of ease of handling, appropriateness in the modeling and/or convenience of numerical implementation. A fair comparison between the quantitative models with these functions should be made to reveal which potential is superior in terms of the numerical performance. This point is addressed in this study.

The interpolating function $g_{q}(\phi)$ in Eq. (4) determines the contribution of the driving force to $\partial_{t} \phi$ in the interface region. Four types of function listed in Table 1 have been employed in previous studies [13-35], and these are tested in this study. All these functions are monotonically increasing functions of $\phi$ in the range of $-1 \leq \phi \leq 1 . g_{1}(\phi)$ has been employed in most quantitative phase-field simulations [13-30]. The usage of $g_{3}(\phi)$ can be found in Ref. [30]. Here, care is necessary in the numerical simulation of the model with $g_{4}(\phi)$ and $f_{1}(\phi)$. When $g_{1}(\phi), g_{2}(\phi)$ and $g_{3}(\phi)$ are used, the contribution of the driving force to $\partial_{t} \phi$ vanishes at $\phi= \pm 1$ because $\mathrm{d} g_{q} / \mathrm{d} \phi=0$ at $\phi= \pm 1$. In the case of $g_{4}(\phi)$, however, the contribution of the driving force is finite even at $\phi= \pm 1$ since $\mathrm{d} g_{4} / \mathrm{d} \phi=1$. 
This does not cause any problem in the model with the double-obstacle potential because the numerical integration of Eq. (4) is prohibited at $\phi= \pm 1$, where $f_{2}(\phi)=\infty$. The finite contribution of the driving force at $\phi= \pm 1$ causes a problem in the model with $f_{1}(\phi)$. Specifically, it results in a large deviation of the $\phi$ profile from Eq. (22). In this case, hence, the contribution of the driving force should be removed near $\phi= \pm 1$. In this study, a cutoff value of the phase-field variable, $\phi_{\mathrm{c}}$, is introduced and Eq. (4) is solved only for the region of $|\phi|<\phi_{\mathrm{c}}$ in the model with $f_{1}(\phi)$ and $g_{4}(\phi)$. Finally, the interpolating function $h_{r}(\phi)$ describes the continuous variations of the concentration and diffusivity in the interface, and we employed the three types of functions shown in Table 1.

In Eq. (10), $a_{A T}(\phi)$ depends on both $f_{p}(\phi)$ and $h_{r}(\phi)$. The form of $a_{A T}(\phi)$ for each set of $f_{p}(\phi)$ and $h_{r}(\phi)$ is shown in Table 2, where $p$ and $r$ indicate the types of functions $f_{p}(\phi)$ and $h_{r}(\phi)$, respectively. Furthermore, the solvability integrals $I, J, H$ and $K$ and the constants $a_{1}$ and $a_{2}$ for each set of functions are listed in Table 3 . All these values were analytically obtained except for the case of $p=1$ and $q=3$, where the value of $K$ was calculated by the numerical integration of Eq. (19). In this study, these models are denoted as models $p$ - $q-r$ as listed in the last column in Table 3. Model 1-1-1 is the quantitative phase-field model employed in most of the previous works [13-29] and its numerical accuracy has been investigated in detail. The quantitative model 2-3-1 was employed in the simulation of directional solidification in Ref. [30] and its convergence behavior was demonstrated in Ref. [31]. In this study, the numerical accuracy of the 24 different models listed in Table 3 is investigated.

\subsection{Thickness measure}

The interface thickness is an important parameter affecting the accuracy of the phase-field models. If the interface is defined as the region of $|\phi|<1$, its thickness is clearly given as $\pi W_{D O}$ in the models with the double-obstacle potential $f_{2}(\phi)$. On the other hand, the thickness cannot be well defined in the models with the double-well potential $f_{1}(\phi)$, as can be understood from the hyperbolic tangent function, Eq. (22). In previous works on model 1-1-1 [13, 15, 19-21, 23], $W_{D W}$ was employed as a measure of the interface thickness in the numerical testing. In this study, we define the following quantity, $W_{c}$, as a measure of the thickness for the models with $f_{1}(\phi)$ and $f_{2}(\phi)$;

$$
W_{c}=W_{D W}=W_{D O} / \sqrt{2} \text {. }
$$

When the same value is assigned to $W_{c}$ in both models, the slopes of $\phi$ at $\phi=0$ are identical in both models. Figure 1(a) shows the steady-state profiles of $\phi$ calculated by Eqs. (22) and (23) for $W_{C}=$ $1 \times 10^{-6} \mathrm{~m}$. Note that the actual interface thickness differs between the models, depending on the definition of the interface region. When the region of $-\phi_{W} \leq \phi \leq \phi_{W}$ is defined as the interface region, the actual interface thickness depends on $\phi_{W}$. The ratio of the actual interface thickness in the model with $f_{1}(\phi)$ to that with $f_{2}(\phi)$ is plotted against $\phi_{W}$ in Fig. 1 (b) where some values are indicated for 
reference. At the same value of $W_{c}$, the actual thickness in the model with $f_{1}(\phi)$ is always larger than that in the model with $f_{2}(\phi)$. In this paper, $W_{c}$ is called the thickness measure to distinguish it from the actual interface thickness, which is defined by $\phi_{W}$ and denoted by $W_{a}$.

\subsection{Computational detail}

We carried out one- and two-dimensional simulations of isothermal solidification in binary dilute alloys. Equations (4) and (5) were discretized on the basis of second-order finite difference formulas with a square grid spacing of $\Delta x$. The time evolutions of the $\phi$ and $u$ fields were solved using a simple first-order Euler scheme as in previous works [19-21]. All simulations were performed using a TESLA K40 graphics processing unit (GPU) [9, 27, 29].

\section{Results and Discussion}

\subsection{One-dimensional analysis of moving flat interface}

We conducted a one-dimensional analysis of a steady-state flat interface moving in an undercooled melt during isothermal solidification. It is known that an analytical solution of the sharp-interface equations (1)-(3) can be obtained for this problem. When Eqs. (1)-(3) are rewritten in a frame moving with a constant interfacial velocity $V_{n}$ along the coordinate $\eta$, the steady-state concentration profile is given as

$$
c=\frac{c_{0}(1-k)}{k} \exp \left(-\frac{V_{n} \eta}{D_{l}}\right)+c_{0}
$$

in the liquid ( $\eta \geq 0$ ) and $c=c_{0}$ in the solid $\left(\eta<0\right.$ ), where $c_{0}$ is the average concentration. The interfacial velocity $V_{n}$ is given as

$$
\beta V_{n}=\frac{\Omega-1}{k},
$$

where $\Omega$ is the initial undercooling defined as $\Omega=-\left(c_{0}-c_{l}^{e}\right) /\left[(1-k) c_{l}^{e}\right]$. The results of the phase-field simulations are compared with the analytical solution.

In the phase-field simulation, we focused on a model alloy system and employed the following parameters: partition coefficient $k=0.2$, chemical capillary length $d_{0}=2 \times 10^{-8} \mathrm{~m}$, liquid diffusivity $D_{l}=2 \times 10^{-8} \mathrm{~m}^{2} / \mathrm{s}$, linear kinetic coefficient $\beta^{-1}=5 \mathrm{~m} / \mathrm{s}$. The time step $\Delta t$ was set to $\Delta t=$ $\Delta x^{2} /\left(3 D_{l}\right)$. The zero-flux boundary condition was applied to both edges of the system. A solid seed was initially placed at the left-hand edge and the solidification proceeded from left to right side. The moving frame was employed to track the position of the interface and the simulations were conducted until steady-state growth was realized.

The spatial grid spacing $\Delta x$ was given by $\Delta x=L_{s y s} / N_{p}$, where $L_{s y s}$ is the system size and $N_{p}$ is the total number of spatial grid points. The thickness measure $W_{c}$ was set to $W_{c}=n_{W} \Delta x$, where $n_{W}$ 
is a constant associated with the number of spatial grid points inside the interface. We investigated the dependence of the accuracy on $n_{W}$ and $\Delta x$ for a fixed value of $W_{c}$. In this test, the accuracy is expected to increase with an increase in $n_{W}$ (thus a decrease in $\Delta x$ ) because the number of spatial grid points inside the interface increases with $n_{W}$ and the variations of $\phi$ and $u$ inside the interface can accordingly be described with high accuracy.

The steady-state profiles of the concentration $c$ near the interface calculated with models 1-1-1 and 2-1-1 are shown in Figs. 2(a) and (b), respectively. In Fig. 2, the concentration $c$ and distance $\eta$ are normalized by $c_{l}{ }^{e}$ and $W_{c}$, respectively. The initial undercooling $\Omega$ was set to $\Omega=1.05$ and the system size $L_{s y s}$ was fixed at $L_{s y s}=8 \times 10^{-7} \mathrm{~m}$. $N_{p}$ was varied from 512 to 2048 and then $n_{W}$ was varied from 1.0 to 4.0. The fixed thickness measure was $W_{c}=1.5625 \times 10^{-9} \mathrm{~m}$. In the phase-field models, the concentration $c$ is given as $c=c_{l}^{e}(1+(1-k) u)(1+k-(1-k) h(\phi)) / 2 . \eta=0$ corresponds to the position of $\phi=0$ in each case. The solid curve indicates the exact solution of Eq. (25). The comparison should be made in the region outside the diffuse interface. The vertical dashed lines indicate the actual interface thickness $W_{a}$ defined by $\phi_{W}=0.95$, which are shown for the convenience of discussion. When $n_{W}=4.0$, both models reproduce the exact solution with high accuracy. However, the concentration profile deviates from the exact solution when $n_{W}=1.0$ in both models. The deviation is significant in model 2-1-1. To evaluate the accuracy in a quantitative manner, the L2 error norm $E_{\mathrm{L} 2}$ was calculated by the following equation [37];

$$
E_{L 2}=\sqrt{\frac{\sum_{k_{p}=1}^{N_{E}}\left(c_{k_{p}}^{p f m}-c_{k_{p}}^{e x}\right)^{2}}{N_{E}}},
$$

where $c_{k_{p}}^{p f m}$ is the concentration at grid point $k_{p}$ calculated by the phase-field simulation, $c_{k_{p}}^{e x}$ is the exact solution of the concentration at point $k_{p}$ and $N_{E}$ is the number of grid points used in the evaluation of $E_{L 2}$. Figure 2(c) shows $E_{L 2}$ calculated in the regions of $\phi<-0.95$ and $\phi>0.95$. We also calculated $E_{L 2}$ for $\phi<-0.99$ and $\phi>0.99$, but the results were not essentially different from those shown in Fig. 2(c). The results for model $p-q-1$ with different sets of $p$ and $q$ are shown. The calculation with model 1-4-1 was performed with a cutoff value of $\phi_{c}=0.01$. Except for model 1-4-1, $E_{L 2}$ for all models monotonically decreases with increasing $n_{W}$. In the case of model 1-4-1, the contribution of the driving force to $\partial_{t} \phi$ does not vanish near $\phi= \pm 1$ as described in Sec. 2.2. This contribution becomes large as $n_{W}$ increases ( $\Delta x$ decreases). Namely, model 1-4-1 is not appropriate in terms of accuracy. Although the results for model 1-4-1 are shown in Figs. 2(d), 3 and 4 for the sake of completeness, the accuracy of this model will not be considered in the following discussion. The important finding from Fig. 2(c) is that the numerical accuracy of the quantitative models is mainly determined by $f_{p}(\phi)$ and that it does not strongly depend on the form of $g_{q}(\phi)$ (except for model 1-4-1). The models with $f_{1}(\phi)$ exhibit relatively high accuracy compared with models with 
$f_{2}(\phi)$. Figure 2(d) shows the dependence of the calculated velocity on $n_{W}$. The calculated velocities of all models gradually converge to the exact solution $\beta V_{n}=0.25$ with increasing $n_{W}$. The convergence of the models with $f_{1}(\phi)$ is faster than that of the models with $f_{2}(\phi)$.

The same analysis was conducted for $\Omega=1.1$ and $L_{s y s}=5 \times 10^{-7} \mathrm{~m} . N_{p}$ was varied from 512 to 2048 and $n_{W}$ was accordingly varied from 1.0 to 4.0. The results are shown in Fig. 3. The same behavior as that in Fig. 2 was observed. More specifically, the accuracy depends on $f_{p}(\phi)$, while it is almost independent of $g_{q}(\phi)$ (except for model 1-4-1). The convergence of the models with $f_{1}(\phi)$ is faster than that of the models with $f_{2}(\phi)$.

As shown in Figs. 2 and 3, the accuracy increases with $n_{W}$. This is because the number of spatial grid points inside the interface increases with $n_{W}$, and thereby the profiles of $\phi$ and $u$ inside the interface can be accurately calculated by employing a large value of $n_{W}$. As mentioned above, the convergence of model 1-q-1 is faster than that of model 2-q-1 in Figs. 2 and 3. In these tests, we assigned the same value to $W_{c}$ in both models. As shown in Fig. 1(b), however, the actual interface thickness $W_{a}$ in the models with $f_{1}(\phi)$ is always larger than that in the models with $f_{2}(\phi)$. Accordingly, the number of spatial grid points in $W_{a}$ is larger in the models with $f_{1}(\phi)$ than in the models with $f_{2}(\phi)$ for the same value of $n_{W}\left(W_{c}\right)$. Therefore, a fair comparison should be made by considering the difference in $W_{a}$. From Figs. 2 and 3, it was found that the results for both models can be approximately merged onto single curves when $W_{a}$ in the models with $f_{1}(\phi)$ is assumed to be about twice as large as that in the models with $f_{2}(\phi)$. This difference in $W_{a}$ corresponds to $\phi_{W} \sim 0.995$ (see Fig. 1(b)). To show this, the results of Figs. 2 and 3 are replotted by doubling the value of $n_{W}$ in the models with $f_{1}(\phi)$ in Fig. 4. It can be seen that the results for the models with $f_{1}(\phi)$ and $f_{2}(\phi)$ are merged onto single curves for each value of $\Omega$. The convergence rate of both models is almost identical in this rescaled plot. To confirm this finding, additional calculations were carried out by using model 2-1-1 for $N_{p}=2048$ and $n_{W}=8$, the results of which are indicated by the diamond symbols at $n_{W}=8$. These results are in good agreement with those calculated by using the models with $f_{1}(\phi)$ for the rescaled $n_{W}=8$ (i.e., $N_{p}=2048$ and $n_{W}=4$ ). Therefore, it can be concluded that the quantitative phase-field models exhibit almost the same accuracy regardless of the choice of $f_{p}(\phi)$ and $g_{q}(\phi)$ in terms of the rescaled $n_{W}$ in the one-dimensional problem.

Although not shown here, the accuracy is slightly dependent on the choice of $h_{r}(\phi)$, particularly in the models with $f_{1}(\phi)$. The accuracy slightly increases as the order of the polynomial decreases, namely, the accuracy is highest in the models with $h_{1}(\phi)$ and lowest in the models with $h_{3}(\phi)$. This should be because $h_{r}(\phi)$ for the high-order polynomial causes rapid variations of the concentration and diffusivity near the center of the interface (i.e., $\phi \sim 0$ ), and the description of such rapid variation requires a small spatial grid spacing. The fact that the accuracy is higher in the models with $h_{1}(\phi)$ than in those with $h_{3}(\phi)$ is consistent with the finding in a previous study on a pure substance with symmetric diffusion [13]. However, it is stressed that the difference in accuracy 
between the models with different $h_{r}(\phi)$ is not substantial.

In these one-dimensional analyses, it was found that the convergence behavior is uniquely characterized using the rescaled $n_{W}$, more precisely, the actual interface thickness $W_{a}$ with $\phi_{W} \sim$ 0.995 for models with $f_{1}(\phi)$ and $f_{2}(\phi)$. Moreover, the quantitative models (except for model 1-4-1) exhibit almost the same numerical efficiency regardless of the choice of $f_{p}(\phi), g_{q}(\phi)$ and $h_{r}(\phi)$. However, in contrast to these findings, the numerical accuracy strongly depends on the choice of $f_{p}(\phi), g_{q}(\phi)$ and $h_{r}(\phi)$ in two-dimensional simulations of dendritic growth as described in detail below.

\subsection{Two-dimensional analysis of isothermal dendritic growth}

In this section, we investigate the numerical accuracy of the quantitative models for isothermal dendritic growth in a two-dimensional system. The migration of a curved interface is involved in this process.

We first carried out a comparison between the calculated shapes of a dendrite for the purpose of rough screening to identify accurate models, which are later subjected to more detailed numerical tests. We focused on a model alloy with $k=0.2, d_{0}=2 \times 10^{-8} \mathrm{~m}, D_{l}=2 \times 10^{-8} \mathrm{~m}^{2} / \mathrm{s}$ and $\beta=$ 0 . The simulations were carried out using a square two-dimensional system with a side length of $L_{\text {sys. }}$. A solid seed with a radius of $1 \times 10^{-6} \mathrm{~m}$ was initially placed at the origin ( $x=0$ and $y=0$ ). The growth of a quarter of a single dendrite was simulated by applying the mirror boundary condition to the edges $x=0$ and $y=0$ and by applying the zero-flux boundary condition to the edges $x=L_{\text {sys }}$ and $y=L_{\text {sys. }}$. The time step $\Delta t$ was set to $\Delta t=\Delta x^{2} /\left(5 D_{l}\right)$. As mentioned in Sec. 2, the numerical integration of Eq. (4) was carried out only for the region of $|\phi|<1$ in the models with $f_{2}(\phi)$ as required from the definition of $f_{2}(\phi)$. On the other hand, no such care is necessary in the models with $f_{1}(\phi)$ and the integration can be performed over the whole system. However, the integration over the whole system wastes the computational cost because $\partial_{t} \phi=0$ at $\phi= \pm 1$ in the models with $f_{1}(\phi)$ (except for models 1-4-r). Hence, Eq. (4) was integrated over only the region of $\left|\phi_{a v}\right| \leq 0.999$ in the models with $f_{1}(\phi)$, where $\phi_{a v}$ represents the average value of $\phi$ over the neighboring grid points. We confirmed that this cutoff does not essentially change the accuracy of the models with $f_{1}(\phi)$ in preliminary simulations.

The shapes of the dendrite calculated with the different models are shown in Fig. 5, where the lines in each figure represent the contour lines of $\phi=0$ at $t=0.114 \mathrm{~s}$ calculated for different values of $N_{p}$. All the calculations were performed for $\Omega=0.2$ and $L_{s y s}=1 \times 10^{-4} \mathrm{~m} . n_{W}$ was fixed at $n_{W}=1.2$, while $\Delta x\left(=L_{s y s} / N_{p}\right)$ and thus $W_{c}\left(=n_{W} \Delta x\right)$ were varied according to $N_{p}$, which was varied from $N_{P}=256^{2}$ to $1536^{2}$. The accuracy is highest in each model when $N_{p}=1536^{2}$ in this test. A cross mark in the legend indicates that the calculation was not stable and the numerical solution was not obtained for the condition. Model $p-q-1$ with different sets of $p$ and $q$ are compared in this figure. When model 1-1-1 is considered, the result does not significantly depend on $N_{p}$. Only the shape 
calculated for $N_{p}=256^{2}$ slightly deviates from those for the other values of $N_{p}$. Therefore, the numerical performance of this model is reasonably high. Such high performance can be also observed in the results for models 2-1-1, 2-2-1 and 2-3-1. However, the simulations with models 1-2-1, 1-3-1 and 2-4-1 are unstable for small values of $N_{p}$ as shown by the cross marks. Also, the result for model 1-4-1 strongly depends on $N_{p}$. Hence, these models are not satisfactory in terms of numerical performance.

The difference in the numerical accuracy between different $g_{q}(\phi)$ becomes marked when the contribution of the driving force in Eq. (4) is large. A similar test to the above was carried out for $\Omega=0.3, L_{\text {sys }}=1.0 \times 10^{-4} \mathrm{~m}$ and $n_{W}=1.2$ and the results are shown in Fig. 6 as the contour lines of $\phi$ $=0$ at $t=0.0305 \mathrm{~s}$. In this case, the numerical solutions for some values of $N_{p}$ were not obtained with models 1-2-1, 1-3-1, 1-4-1, 2-3-1 and 2-4-1. Moreover, the shape calculated for $N_{p}=256^{2}$ markedly deviates from that for $N_{p}=1536^{2}$ in model 2-2-1. On the other hand, models 1-1-1 and 2-1-1 reproduce accurate shapes even for small values of $N_{p}$. Therefore, the present numerical test reveals that the function $g_{1}(\phi)$ is the most appropriate choice for models with both $f_{1}(\phi)$ and $f_{2}(\phi)$. We accordingly focus on the models with $g_{1}(\phi)$ in the following convergence tests.

To investigate the accuracy of the different models in a more quantitative manner, we examined the convergence behavior in the steady-state growth of a dendrite with respect to the spatial grid spacing $\Delta x$. In this convergence test, we employed a rectangular computational box with $L_{x}=L_{s y s}$ and $L_{y}=L_{\text {sys }} / 2$, where $L_{x}$ and $L_{y}$ are the lengths in the $x$ and $y$ directions, respectively. In this test, we fixed $L_{s y s}$ and $n_{W}$, while we varied $W_{c}$ and $\Delta x$ by changing $N_{p}$. The mirror boundary condition was applied to the edge $y=0$ and the zero-flux boundary condition was applied to the other edges. We focused on the same model alloy as before, i.e., the alloy with $k=0.2, d_{0}=2 \times 10^{-8} \mathrm{~m}$, $D_{l}=2 \times 10^{-8} \mathrm{~m}^{2} / \mathrm{s}$ and $\beta=0$. The simulations started from a small solid quarter disk with an initial radius of $1 \times 10^{-6} \mathrm{~m}$ at the origin. To simulate the steady-state growth within a reasonable computational time, a moving-frame calculation was conducted by moving the computational box with a certain velocity in the $x$ direction. In this convergence test, the accuracy of models 1-1-r and 2-1-r with $r=1,2$ and 3 is discussed.

The dependence of the results on the spatial grid spacing $\Delta x$ is shown in Fig. 7, where $\Delta x$ is normalized by the capillary length $d_{0}$. These results were calculated for $\Omega=0.3, L_{s y s}=4 \times 10^{-4} \mathrm{~m}$ and $n_{W}=1.25$. Since $n_{W}$ is fixed in this test, the thickness measure $W_{c}\left(=n_{W} \Delta x\right)$ changes with the value of $\Delta x$. Figure 7 shows the steady-state values of the dendrite tip velocity $V_{n}$ (Fig. 7(a)), the curvature radius of the tip $\rho$ (Fig. 7(b)), the solid composition at $y=0$ far behind the dendrite tip $c_{s}{ }^{*}$ (Fig. 7(c)) and the error in the Gibbs-Thomson relation $\Delta E_{\mathrm{G}}$ (Fig. 7(d)). The curvature radius $\rho$ was calculated by fitting the $\phi=0$ contour at the dendrite tip with a parabola. The error in the Gibbs-Thomson relation $\Delta E_{\mathrm{G}}$ was calculated by the following equation; 


$$
\Delta E_{G}=\frac{c_{s}^{*}}{c_{l}^{e}}-k\left(1-(1-k) \frac{d_{0}\left(1-15 \varepsilon_{4}\right)}{\rho}\right)
$$

In this convergence test, the accuracy should be highest for the smallest value of $\Delta x$, and the fast convergence of the results with decreasing $\Delta x$ indicates good performance. When $V_{n}$ and $\rho$ are considered, all the models predict the same values at the smallest value of $\Delta x$. However, $V_{n}$ and $\rho$ rapidly and markedly deviate from the converged values with increasing $\Delta x$ in models $p-1-2$ and $p-1-3$. The plots of models $p-1-2$ and $p-1-3$ are missing for large values of $\Delta x$ because stable solutions were not obtained for these values of $\Delta x$. On the other hand, solutions for all values of $\Delta x$ were obtained with models 1-1-1 and 2-1-1. Note that models 1-1-1 and 2-1-1 yield almost the same convergence behavior of $V_{n}$ and $\rho$ in Fig. 7. However, a difference in their accuracy appears in $c_{s}{ }^{*}$ (Fig. 7(c)) and $\Delta E_{\mathrm{G}}$ (Fig. 7(d)). $c_{s}^{*}$ takes an almost constant value for $\Delta x / d_{0}<20$ in model 1-1-1, while it still varies in model 2-1-1. The absolute values of $\Delta E_{\mathrm{G}}$ for small values of $\Delta x$ are always smaller in model 1-1-1 than in model 2-1-1. Therefore, the convergence of model 1-1-1 is slightly faster than that of model 2-1-1. Similar results were found in the case of a large degree of undercooling. Figure 8 shows the results of the convergence test for $\Omega=0.5$. The convergence of models $p-1-2$ and $p-1-3$ is rather poor. Models 1-1-1 and 2-1-1 exhibit comparably good convergence behavior of $V_{n}$ and $\rho$. However, the convergence is faster in model 1-1-1 than in model 2-1-1 when $c_{s}^{*}$ and $\Delta E_{\mathrm{G}}$ are considered.

The accuracy of the simulations of dendritic growth should be determined by two factors. The first one is the number of spatial grid points inside the interface. As the number of spatial grid points inside the interface increases (i.e., $n_{W}$ increases), the profiles of $\phi$ and $u$ inside the interface can be calculated more accurately. Hence, the accuracy of results should be improved by increasing $n_{W}$ as discussed for the one-dimensional problem (Fig. 4). The second factor is the resolution capability of describing the shape of the dendrite. In the diffuse interface approach, the interface thickness determines the minimum radius of the dendrite tip that can be accurately described in the simulation. When the interface thickness is comparable to or larger than the tip radius, the interface regions overlap near the tip and hence the shape of the tip cannot be accurately described. Namely, the accurate description of a small curvature radius requires a small interface thickness (i.e., high resolution). To make this point clearer, simulations were carried out for different values of $n_{W}$. The results for $n_{W}=1.10,1.25$ and 1.50 are shown in Fig. 9, where only the best models 1-1-1 and 2-1-1 are compared. The parameters used in the simulations were the same as those in Fig. 8 except for $n_{W}$ and thus $W_{c}$. Since $W_{a}$ is larger in model 1-1-1 than in model 2-1-1 for the same value of $n_{W}$, the simulation was carried out for $n_{W}=2.0$ with model 2-1-1. In both models, the convergence starts to break down for small values of $\Delta x$ when $n_{W}$ is large. This is because the interface thickness increases with $n_{W}$ at a given value of $\Delta x$, which lowers the resolution capability. 
It is convenient to examine the convergence with respect to $\Delta x$ as in Figs. 7-9 to understand the relationship between the accuracy and computational cost. On the other hand, the convergence behavior should be more effectively characterized in terms of $W_{c}$ or $W_{a}$ when the resolution capability is considered. In Fig. 10, all the data in Fig. 9 are replotted against the thickness measure $W_{c}$. All the data of $V_{n}$ and $\rho$ are merged onto single curves, which are independent of $n_{W}$. This indicates that both models can predict $V_{n}$ and $\rho$ with the same accuracy as long as the same value is assigned to $W_{c}$. When $c_{s}{ }^{*}$ and $\Delta E_{\mathrm{G}}$ are considered, the convergence of model 1-1-1 only slightly varies with $n_{W}$, while the convergence strongly depend on $n_{W}$ in model 2-1-1. $c_{s}^{*}$ for model 2-1-1 becomes close to $c_{s}{ }^{*}$ for model 1-1-1 with increasing $n_{W}$, and also $\Delta E_{\mathrm{G}}$ in model 2-1-1 approaches zero for small values of $W_{c}$ with increasing $n_{W}$. Note that the increase in $n_{W}$ increases the computational cost for a fixed value of $W_{c}$ because the small grid spacing $\Delta x=W_{c} / n_{W}$ is required. Hence, model 1-1-1 is slightly superior to model 2-1-1 in terms of numerical efficiency.

It is important to point out that the accuracy of models 1-1-1 and 2-1-1 shown in Figs. 7-9 is significantly higher than that of the standard models. As demonstrated for model 1-1-1 in Refs. [19, 21], $c_{s}{ }^{*}$ for the standard models (the models without the anti-trapping current) is strongly dependent on $W_{c}$ and the resulting $\Delta E_{\mathrm{G}}$ is more than one order of magnitude larger than the values shown in Figs. 7-10. Namely, the differences in $c_{s}{ }^{*}$ and $\Delta E_{\mathrm{G}}$ between models 1-1-1 and 2-1-1 are small compared with the differences between the quantitative and standard models. Therefore, it is concluded that models 1-1-1 and 2-1-1 exhibit comparably good numerical performance.

Although the results for only models 1-1-r and 2-1-r with $r=1,2$ and 3 were shown in Figs. 7 and 8, the convergence tests were carried out for all the models with different sets of $f_{p}(\phi)$, $g_{q}(\phi)$ and $h_{r}(\phi)$ in this study. It was found that the accuracy of the model with $h_{r}(\phi) \neq h_{1}(\phi)$ is always much lower than that of the models with $h_{1}(\phi)$ for any set of $f_{p}(\phi)$ and $g_{q}(\phi)$. Also, the calculations of the models with $f_{1}(\phi)$ and $g_{q}(\phi) \neq g_{1}(\phi)$ were unstable under most of the computational conditions used in Figs. 7 and 8. Even if a solution was obtained, the accuracy was always much lower than that of model 1-1-1. Among the models with $f_{2}(\phi)$ and $g_{q}(\phi) \neq g_{1}(\phi)$, models 2-2-1 and 2-3-1 yield reasonable accuracy as can be seen from Figs. 5 and 6. However, the accuracy of models 2-2-1 and 2-3-1 is always lower than that of model 2-1-1 in the convergence tests on steady-state growth. Hence, it was confirmed that the set of $g_{1}(\phi)$ and $h_{1}(\phi)$ is the best choice for models with both $f_{1}(\phi)$ and $f_{2}(\phi)$. However, with the exception of models 1-1-1 and 2-1-1, the numerical accuracy of models 2-2-1 and 2-3-1 is higher than that of the other models. It is expected that these models can exhibit reasonable numerical performance for some solidification conditions (e.g., a low degree of undercooling) as demonstrated for model 2-3-1 in Ref. [31]. In this regard, compared with $f_{1}(\phi)$, the model with $f_{2}(\phi)$ may offer more choices in constructing a quantitative phase-field model with reasonable numerical performance, which is beneficial for extending the range of applications of quantitative simulations. 
Finally, let us discuss a unique aspect of the convergence behavior related to the resolution capability. By comparing Figs. 7-9, one notices that the critical value of $\Delta x$ (and thus the critical value of $W_{c}$ ) at which the convergence starts to break down is strongly dependent on the solidification condition $\Omega$. In practice, therefore, the value of $\Delta x\left(W_{c}\right)$ necessary for accurate simulation must be found by carrying out burdensome convergence tests for each condition of interest. However, as discussed in Ref. [10], the convergence behavior of $V_{n}$ and $\rho$ in model 1-1-1 can be uniquely characterized on normalized scales regardless of the solidification condition and alloy system. The convergence of model 2-1-1 can be uniquely characterized in the same way. In Fig. 11, all the data of $V_{n}$ and $\rho$ in Figs. 7 and 9 are plotted on normalized scales, where $V_{n}$ and $\rho$ are normalized by $V_{c}$ and $\rho_{c}$, respectively, and $W_{c}$ is normalized by $\rho_{c}$. Here, $V_{c}$ and $\rho_{c}$ are the converged values of $V_{n}$ and $\rho$, respectively, that correspond to those calculated for the smallest value of $\Delta x$ for each value of $\Omega$. The results of Refs. [19, 21] are shown for reference. As $W_{c}$ increases, $V_{n}$ decreases and $\rho$ increases in all the cases. Importantly, the results of the present simulations including those with model 2-1-1 exhibit unique convergence behavior regardless of $\Omega$ and they are almost coincident with the data in the previous studies. The convergence starts to break down for $W_{c} / \rho_{c} \sim$ 0.2 in all the cases. In model 1-1-1, this condition corresponds to the condition of $W_{a} \sim \rho_{c}$ with $W_{a}$ defined by $\phi_{W}=0.95$ [10]. In the case of model 2-1-1, $W_{a}=\pi W_{D O}=\pi(2)^{1 / 2} W_{c}$ for $\phi_{W}=1$, and hence the condition $W_{c} / \rho_{c} \sim 0.2$ is approximately equivalent to the condition $W_{a} \sim \rho_{c}$. These facts indicate that the breakdown of the convergence of $V_{n}$ and $\rho$ stems from the limitation due to the resolution capability in both models. Hence, the unphysical magnification of interface effects, which is a critical problem of the standard models, is sufficiently suppressed in models 1-1-1 and 2-1-1. The result shown in Fig. 11 should be useful for reducing the effort required to find a suitable value of $W_{c}$ for accurate simulations.

\section{Conclusions}

Quantitative phase-field models have been developed as effective tools for solving the free-boundary problem [13, 14]. The numerical efficiency of these quantitative models depends on the set of interpolating functions that represent the continuous variations of the physical quantities inside the interface. In this paper, the numerical accuracy of 24 different models was investigated in detail by carrying out one-dimensional simulations of a moving flat interface and two-dimensional simulations of the dendritic growth during isothermal solidification in dilute binary alloys. In the one-dimensional problem, the accuracy of the simulations is essentially independent of the choice of the interpolating functions (except for model 1-4-1). However, in the case of the two-dimensional problem, where the curved interface migrates, the set of interpolating functions should carefully be chosen to achieve high numerical accuracy. In both models with double-well and double-obstacle potentials, high numerical accuracy can be achieved by employing the fifth-order and first-order 
polynomials, i.e., $g_{1}(\phi)$ and $h_{1}(\phi)$, as the interpolating functions of the driving force and concentration changes inside the interface, respectively. Quantitative phase-field models have so far been developed for only some specific physical systems [15-21, 30, 32], and further extension of the range of applications of the quantitative models is required to realize accurate control and analysis of a variety of solidification microstructures. We expect that the present findings should be useful for the development of highly accurate quantitative phase-field models with extended ranges of applications.

\section{Acknowledgements}

This research was supported by Grant-in-Aid for Scientific Research (B) (JSPS KAKENHI Grant Number 16H04541) from Japan Society for the Promotion of Science (JSPS), the High Performance Computing Infrastructure (HPCI) in Japan. Also, this work was supported in part by MEXT as a social and scientific priority issue (Creation of new functional devices and high-performance materials to support next-generation industries) to be tackled by using post-K computer.

\section{References}

[1] Y.-T. Kim, N. Goldenfeld and J. Dantzig. Computation of dendritic microstructures using a level set method, Phys. Rev. E 62 (2000) 2471-2474.

[2] F. Gibou, R. Fedkiw, R. Caflisch and S. Osher, A Level Set Approach for the Numerical Simulation of Dendritic Growth, J. Sci. Comput. 19 (2003) 183-199.

[3] H. Chen, C. Min, F. Gibou, A numerical scheme for the Stefan problem on adaptive Cartesian grids with supralinear convergence rate, J. Comput. Phys. 228 (2009) 5803-5818.

[4] M. Theillard, F. Gibou and T. Pollock, A Sharp Computational Method for the Simulation of the Solidification of Binary Alloys, J. Sci. Comput. 63 (2015) 330-354.

[5] R. Kobayashi, Modeling and numerical simulations of dendritic crystal growth, Physica D 63 (1993) 410-423.

[6] N. Provatas and K. Elder, Phase-field methods in materials science and engineering, 2010, Wiley-VCH.

[7] I. Steinbach, Phase-field models in materials science, Modell. Simul. Mater. Sci. Eng. 17 (2009) 073001.

[8] T. Takaki, Phase-field Modeling and Simulations of Dendrite Growth, ISIJ Int. 54 (2014) 437-444.

[9] T. Takaki, T. Shimokawabe, M. Ohno, A. Yamanaka and T. Aoki, Unexpected selection of growing dendrites by very-large-scale phase-field simulation, J. Cryst. Growth 382 (2013) 21-25.

[10] Y. Shibuta, M. Ohno and T. Takaki, Solidification in a Supercomputer: From Crystal Nuclei to 
Dendrite Assemblages, JOM 67 (2015) 1793-1804.

[11] Y. Shibuta, K. Oguchi and M. Ohno, Million-atom molecular dynamics simulation on spontaneous evolution of anisotropy in solid nucleus during solidification of iron, Scr. Mater. 86 (2014) 20-23.

[12] M. Asta, C. Beckermann, A. Karma, W. Kurz, R. Napolitano, M. Plapp, G. Purdy, M. Rappaz, and R. Trivedi, Solidification microstructures and solid-state parallels: Recent developments, future directions, Acta Mater. 57 (2009) 941-971.

[13] A. Karma and W.-J. Rappel, Quantitative phase-field modeling of dendritic growth in two and three dimensions, Phys. Rev. E 57 (1998) 4323-4349.

[14] A. Karma, Phase-Field Formulation for Quantitative Modeling of Alloy Solidification, Phys. Rev. Lett. 87 (2001) 115701.

[15] B. Echebarria, R. Folch, A. Karma and M. Plapp, Quantitative phase-field model for alloy solidification, Phys. Rev. E 70 (2004) 061604.

[16] J.C. Ramirez, C. Beckermann, A. Karma, and H.-J. Diepers, Phase-field modeling of binary alloy solidification with coupled heat and solute diffusion, Phys. Rev. E 69 (2004) 051607.

[17] R. Folch and M. Plapp, Quantitative phase-field modeling of two-phase growth, Phys. Rev. E 72 (2005) 011602.

[18] S.G. Kim, A phase-field model with antitrapping current for multicomponent alloys with arbitrary thermodynamic properties, Acta Mater. 55 (2007) 4391-4399.

[19] M. Ohno and K. Matsuura, Quantitative phase-field modeling for dilute alloy solidification involving diffusion in the solid, Phys. Rev. E 79 (2009) 031603.

[20] M. Ohno and K. Matsuura, Quantitative phase-field modeling for two-phase solidification process involving diffusion in the solid, Acta Mater. 58 (2010) 5749-5758.

[21] M. Ohno, Quantitative phase-field modeling of nonisothermal solidification in dilute multicomponent alloys with arbitrary diffusivities, Phys. Rev. E 86 (2012) 051603.

[22] G. Boussinot and E.A. Brener, Achieving realistic interface kinetics in phase-field models with a diffusional contrast, Phys. Rev. E 89 (2014) 060402 (R).

[23] M. Ohno, T. Takaki and Y. Shibuta, Variational formulation and numerical accuracy of a quantitative phase-field model for binary alloy solidification with two-sided diffusion, Phys. Rev. E 93 (2016) 012802.

[24] M. Greenwood, M. Haataja and N. Provatas, Crossover Scaling of Wavelength Selection in Directional Solidification of Binary Alloys, Phys. Rev. Lett. 93 (2004) 246101.

[25] S. Gurevich, A. Karma, M. Plapp, R. Trivedi, Phase-field study of three-dimensional steady-state growth shapes in directional solidification, Phys. Rev. E 81 (2010) 011603.

[26] Z. Wang, J. Li, J. Wang and Y. Zhou, Phase field modeling the selection mechanism of primary dendritic spacing in directional solidification, Acta Mater. 60 (2012) 1957-1964. 
[27] T. Takaki, M. Ohno, T. Shimokawabe and T. Aoki, Two-dimensional phase-field simulations of dendrite competitive growth during the directional solidification of a binary alloy bicrystal, Acta Mater. 81 (2014) 272-283.

[28] R. Rojas, T. Takaki, M. Ohno, A phase-field-lattice Boltzmann method for modeling motion and growth of a dendrite for binary alloy solidification in the presence of melt convection, J. Comput. Phys. 298 (2015) 29-40.

[29] T. Takaki, M. Ohno, Y. Shibuta, S. Sakane, T. Shimokawabe, T. Aoki, Two-dimensional phase-field study of competitive growth during directional solidification of polycrystalline binary alloy, J. Cryst. Growth 442 (2016) 14-24.

[30] I. Steinbach, Effect of interface anisotropy on spacing selection in constrained dendrite growth, Acta Mater. 56 (2008) 4965-4971.

[31] I. Steinbach, Why Solidification? Why Phase-Field?, JOM 65 (2013) 1096-1102.

[32] A. Choudhury and B. Nestler, Grand-potential formulation for multicomponent phase transformations combined with thin-interface asymptotics of the double-obstacle potential, Phys. Rev. E 85 (2012) 021602.

[33] I. Steinbach, F. Pezzolla, A generalized field method for multiphase transformations using interface fields, Physica D 134 (1999) 385-393.

[34] S.G. Kim, W.T. Kim, T. Suzuki, M. Ode, Phase-field modeling of eutectic solidification, J. Cryst. Growth 261 (2004) 135-158.

[35] J. Eiken, B. Böttger and I. Steinbach, Multiphase-field approach for multicomponent alloys with extrapolation scheme for numerical application, Phys. Rev. E 73 (2006) 066122.

[36] B. Nestler, A.A. Wheeler and H. Garcke, Modelling of microstructure formation and interface dynamics, Comp. Mater. Sci. 26 (2003) 111-119.

[37] Y. Sun and C. Beckermann, Sharp interface tracking using the phase-field equation, J. Comput. Phys. 220 (2007) 626-653. 
Table 1. Interpolating functions employed in this study (left-hand side) and their first derivatives (right-hand side).

\begin{tabular}{|c|c|c|c|}
\hline \multicolumn{4}{|c|}{$f_{p}$} \\
\hline$f_{1}$ & $\frac{1}{4}\left(1-\phi^{2}\right)^{2}$ & $\mathrm{~d} f_{1} / \mathrm{d} \phi$ & $-\phi+\phi^{3}$ \\
\hline$f_{2}$ & $\frac{1}{2}\left(1-\phi^{2}\right)$ & $\mathrm{d} f_{2} / \mathrm{d} \phi$ & $-\phi$ \\
\hline \multicolumn{4}{|c|}{$g_{q}$} \\
\hline$g_{1}$ & $\frac{15}{8}\left(\phi-\frac{2}{3} \phi^{3}+\frac{\phi^{5}}{5}\right)$ & $\mathrm{d} g_{1} / \mathrm{d} \phi$ & $\frac{15}{8}\left(1-\phi^{2}\right)^{2}$ \\
\hline$g_{2}$ & $\frac{1}{2}\left(3 \phi-\phi^{3}\right)$ & $\mathrm{d} g_{2} / \mathrm{d} \phi$ & $\frac{3}{2}\left(1-\phi^{2}\right)$ \\
\hline$g_{3}$ & $\frac{2}{\pi}\left(\sin ^{-1} \phi+\phi \sqrt{1-\phi^{2}}\right)$ & $\mathrm{d} g_{3} / \mathrm{d} \phi$ & $\frac{4}{\pi} \sqrt{1-\phi^{2}}$ \\
\hline$g_{4}$ & $\phi$ & $\mathrm{d} g_{4} / \mathrm{d} \phi$ & 1 \\
\hline \multicolumn{4}{|c|}{$h_{r}$} \\
\hline$h_{1}$ & $\phi$ & $\mathrm{d} h_{1} / \mathrm{d} \phi$ & 1 \\
\hline$h_{2}$ & $\frac{1}{2}\left(3 \phi-\phi^{3}\right)$ & $\mathrm{d} h_{2} / \mathrm{d} \phi$ & $\frac{3}{2}\left(1-\phi^{2}\right)$ \\
\hline$h_{3}$ & $\frac{15}{8}\left(\phi-\frac{2}{3} \phi^{3}+\frac{\phi^{5}}{5}\right)$ & $\mathrm{d} h_{3} / \mathrm{d} \phi$ & $\frac{15}{8}\left(1-\phi^{2}\right)^{2}$ \\
\hline
\end{tabular}


Table 2. Coefficient of antitrapping current, $a_{\mathrm{AT}}(\phi)$.

\begin{tabular}{l|c|l}
\hline \hline$p$ & $r$ & \\
\hline & 1 & $\frac{1}{2 \sqrt{2}}$ \\
\cline { 2 - 4 } & 2 & $\frac{1}{8 \sqrt{2}}\left(\phi^{2}-1\right)\left(\phi^{2}-4\right)$ \\
\cline { 2 - 4 } & 3 & $\frac{1}{128 \sqrt{2}}\left(8-7 \phi(1+\phi)+3 \phi^{3}(1+\phi)\right)\left(8+7 \phi(1-\phi)-3 \phi^{3}(1-\phi)\right)$ \\
\hline \multirow{2}{*}{2} & 2 & $\frac{1}{4} \sqrt{\left(1-\phi^{2}\right)}$ \\
\cline { 2 - 4 } & 1 & $\frac{1}{\left(1-\phi^{2}\right)}\left(\phi^{2}-1\right)\left(\phi^{2}-4\right)$ \\
\cline { 2 - 4 } & 3 & $\frac{1}{256} \sqrt{\left(1-\phi^{2}\right)}\left(8-7 \phi(1+\phi)+3 \phi^{3}(1+\phi)\right)\left(8+7 \phi(1-\phi)-3 \phi^{3}(1-\phi)\right)$ \\
\hline
\end{tabular}


Table 3. Values for the solvability integrals and constants for each quantitative phase-field model.

\begin{tabular}{|c|c|c|c|c|c|c|c|c|c|}
\hline$p$ & $q$ & $r$ & $I$ & $J$ & $H$ & $K$ & $a_{1}$ & $a_{2}$ & model \\
\hline \multirow{12}{*}{1} & \multirow{3}{*}{1} & 1 & \multirow{12}{*}{0.94281} & \multirow{3}{*}{1.0667} & 0.98026 & 0.13605 & \multirow{3}{*}{0.88388} & 0.62667 & $1-1-1$ \\
\hline & & 2 & & & 0.62671 & 0.18992 & & 0.45524 & $1-1-2$ \\
\hline & & 3 & & & 0.49412 & 0.22359 & & 0.39810 & $1-1-3$ \\
\hline & \multirow{3}{*}{2} & 1 & & \multirow{3}{*}{1.3333} & 0.98026 & 0.26434 & \multirow{3}{*}{0.70711} & 0.83333 & $1-2-1$ \\
\hline & & 2 & & & 0.62671 & 0.35862 & & 0.63333 & $1-2-2$ \\
\hline & & 3 & & & 0.49412 & 0.41418 & & 0.56905 & $1-2-3$ \\
\hline & \multirow{3}{*}{3} & 1 & & \multirow{3}{*}{1.5708} & 0.98026 & $0.42907 *$ & \multirow{3}{*}{0.60021} & 1.0441 & $1-3-1$ \\
\hline & & 2 & & & 0.62671 & $0.56791 *$ & & 0.82325 & $1-3-2$ \\
\hline & & 3 & & & 0.49412 & $0.64600 *$ & & 0.75422 & $1-3-3$ \\
\hline & \multirow{3}{*}{4} & 1 & & \multirow{3}{*}{2.0000} & 0.98026 & 0.86791 & \multirow{3}{*}{0.47140} & 1.5000 & $1-4-1$ \\
\hline & & 2 & & & 0.62671 & 1.1036 & & 1.2500 & $1-4-2$ \\
\hline & & 3 & & & 0.49412 & 1.2274 & & 1.1750 & $1-4-3$ \\
\hline \multirow{12}{*}{2} & \multirow{3}{*}{1} & 1 & \multirow{12}{*}{1.5708} & \multirow{3}{*}{1.0667} & 0.57080 & 0.08492 & \multirow{3}{*}{1.47262} & 0.22083 & 2-1-1 \\
\hline & & 2 & & & 0.40413 & 0.11953 & & 0.17526 & $2-1-2$ \\
\hline & & 3 & & & 0.32913 & 0.14154 & & 0.15680 & $2-1-3$ \\
\hline & \multirow{3}{*}{2} & 1 & & \multirow{3}{*}{1.3333} & 0.57080 & 0.15524 & \multirow{3}{*}{1.17810} & 0.29167 & $2-2-1$ \\
\hline & & 2 & & & 0.40413 & 0.21383 & & 0.23958 & $2-2-2$ \\
\hline & & 3 & & & 0.32913 & 0.24941 & & 0.21908 & $2-2-3$ \\
\hline & \multirow{3}{*}{3} & 1 & & \multirow{3}{*}{1.5708} & 0.57080 & 0.23746 & \multirow{3}{*}{1.00000} & 0.36098 & $2-3-1$ \\
\hline & & 2 & & & 0.40413 & 0.32149 & & 0.30440 & $2-3-2$ \\
\hline & & 3 & & & 0.32913 & 0.37072 & & 0.28257 & $2-3-3$ \\
\hline & \multirow{3}{*}{4} & 1 & & \multirow{3}{*}{2.0000} & 0.57080 & 0.42920 & \multirow{3}{*}{0.78540} & 0.50000 & $2-4-1$ \\
\hline & & 2 & & & 0.40413 & 0.56619 & & 0.43750 & $2-4-2$ \\
\hline & & 3 & & & 0.32913 & 0.64256 & & 0.41406 & $2-4-3$ \\
\hline
\end{tabular}

* These values were obtained by numerical integration. 
(a)

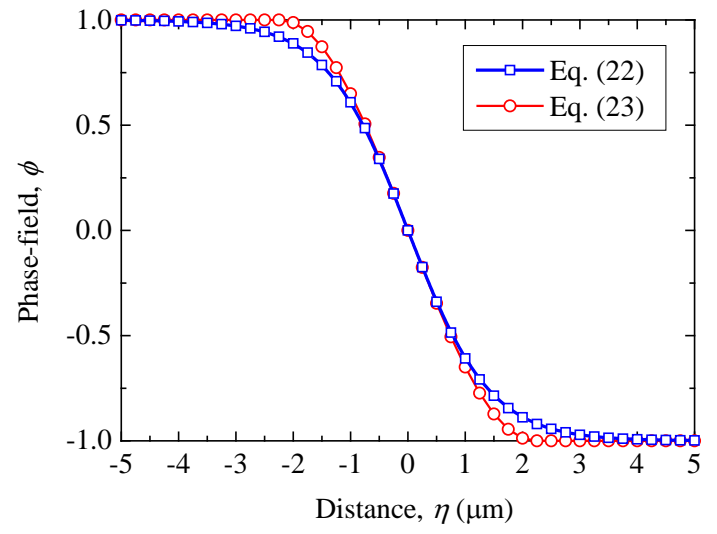

(b)

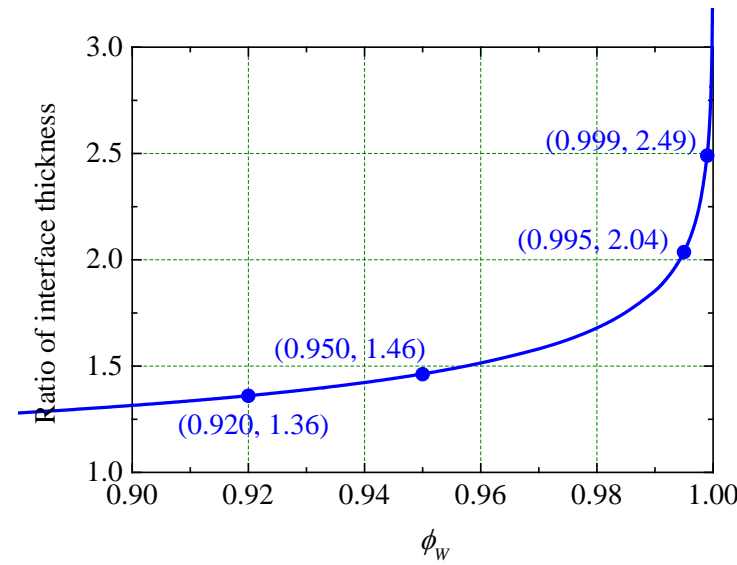

Fig. 1. (a) Phase-field profiles calculated by Eqs. (22) and (23) for $W_{C}=1 \times 10^{-6} \mathrm{~m}$. (b) Ratio of interface thickness of model 1-q-r to that of model 2-q-r plotted against the cutoff value of the phase-field variable, $\phi_{w}$. 
(a)

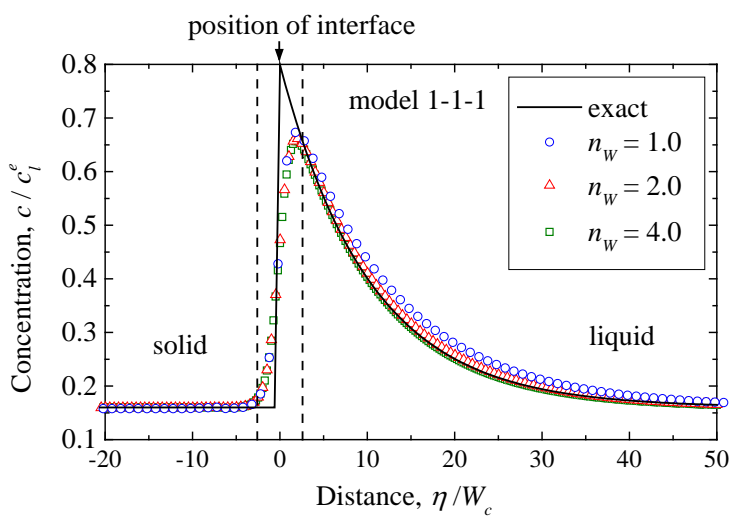

(c)

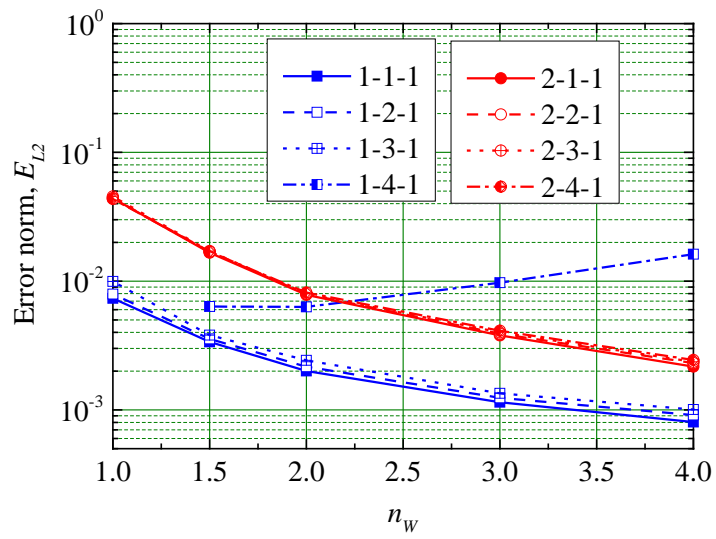

(b)

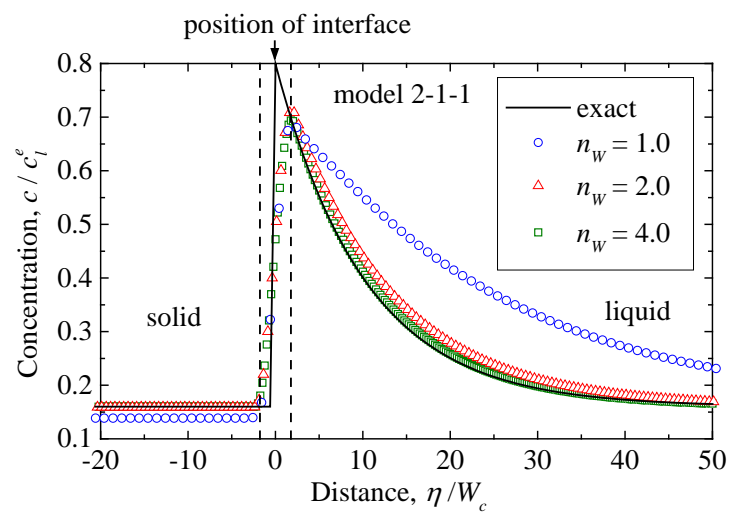

(d)

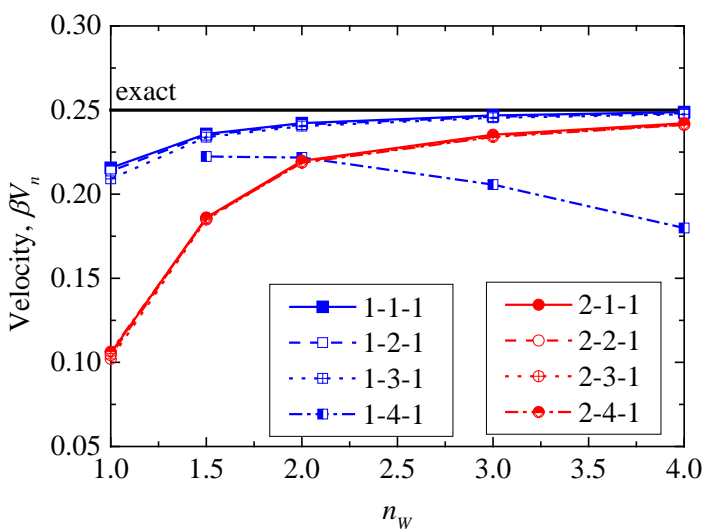

Fig. 2. Results of $1 \mathrm{D}$ simulations for $\Omega=1.05$. (a, b) Concentration profiles near the interface calculated by (a) model 1-1-1 and (b) model 2-1-1. The vertical dashed lines represent the edges of the interface region defined by $\phi_{W}=0.95$. (c) Dependence of L2 error norm on $n_{W}$. (d) Dependence of interface velocity on $n_{W}$. In (c) and (d), the plots for models 2-q-1 almost overlap with each other. 
(a)

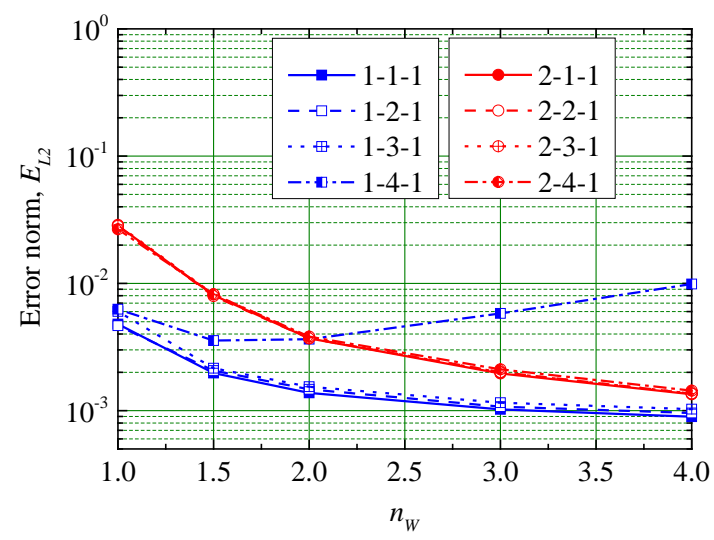

(b)

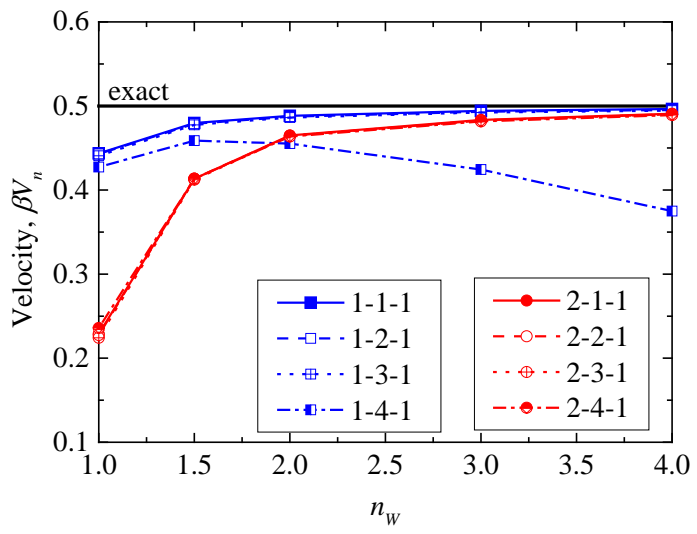

Fig. 3. Results of $1 \mathrm{D}$ simulations for $\Omega=1.1$. (a) Dependence of L2 error norm on $n_{W}$. (b) Dependence of interface velocity on $n_{W}$. In (a) and (b), the plots for model 2-q-1 almost overlap with each other. 
(a)

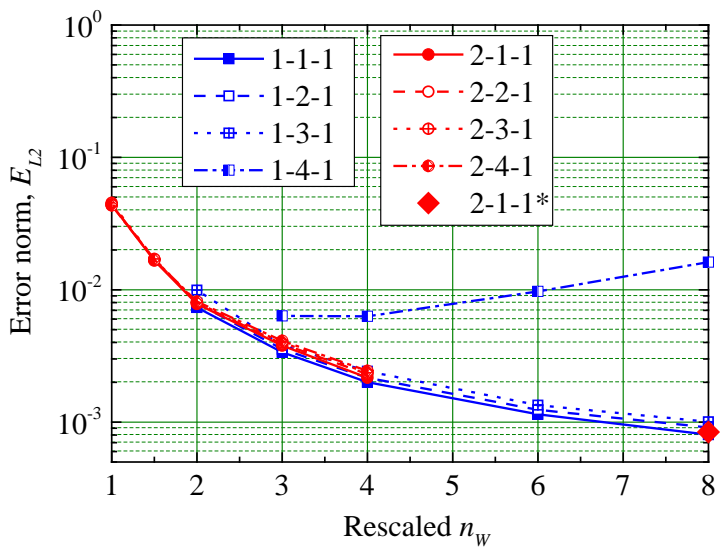

(c)

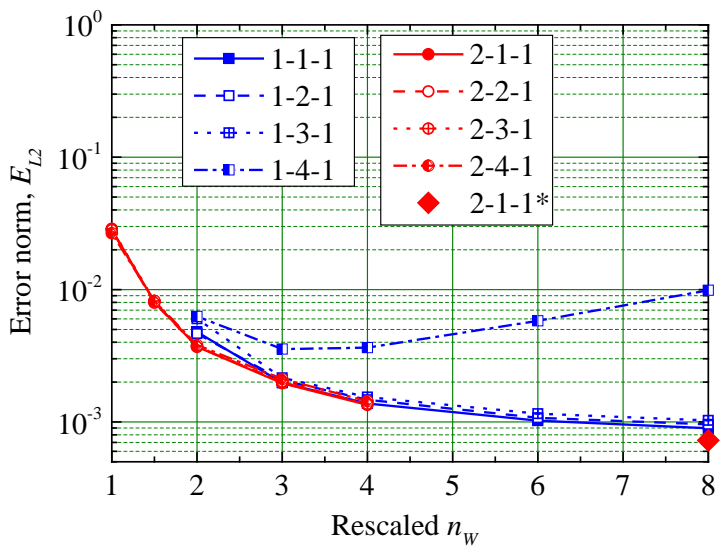

(b)

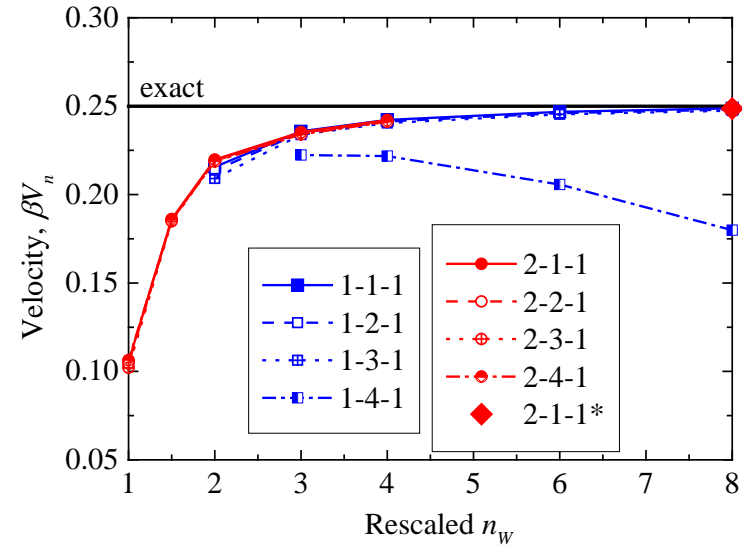

(d)

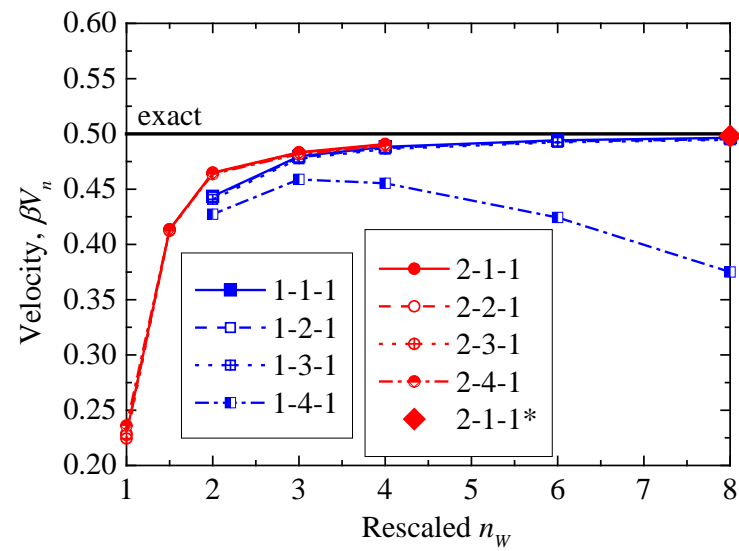

Fig. 4. (a, b) Results for $\Omega=1.05$, indicating dependence of (a) $E_{L 2}$ and (b) $\beta V_{n}$ on rescaled $n_{W}$. (c, d) Results for $\Omega=1.1$, indicating dependence of (c) $E_{L 2}$ and (d) $\beta V_{n}$ on rescaled $n_{W}$. 


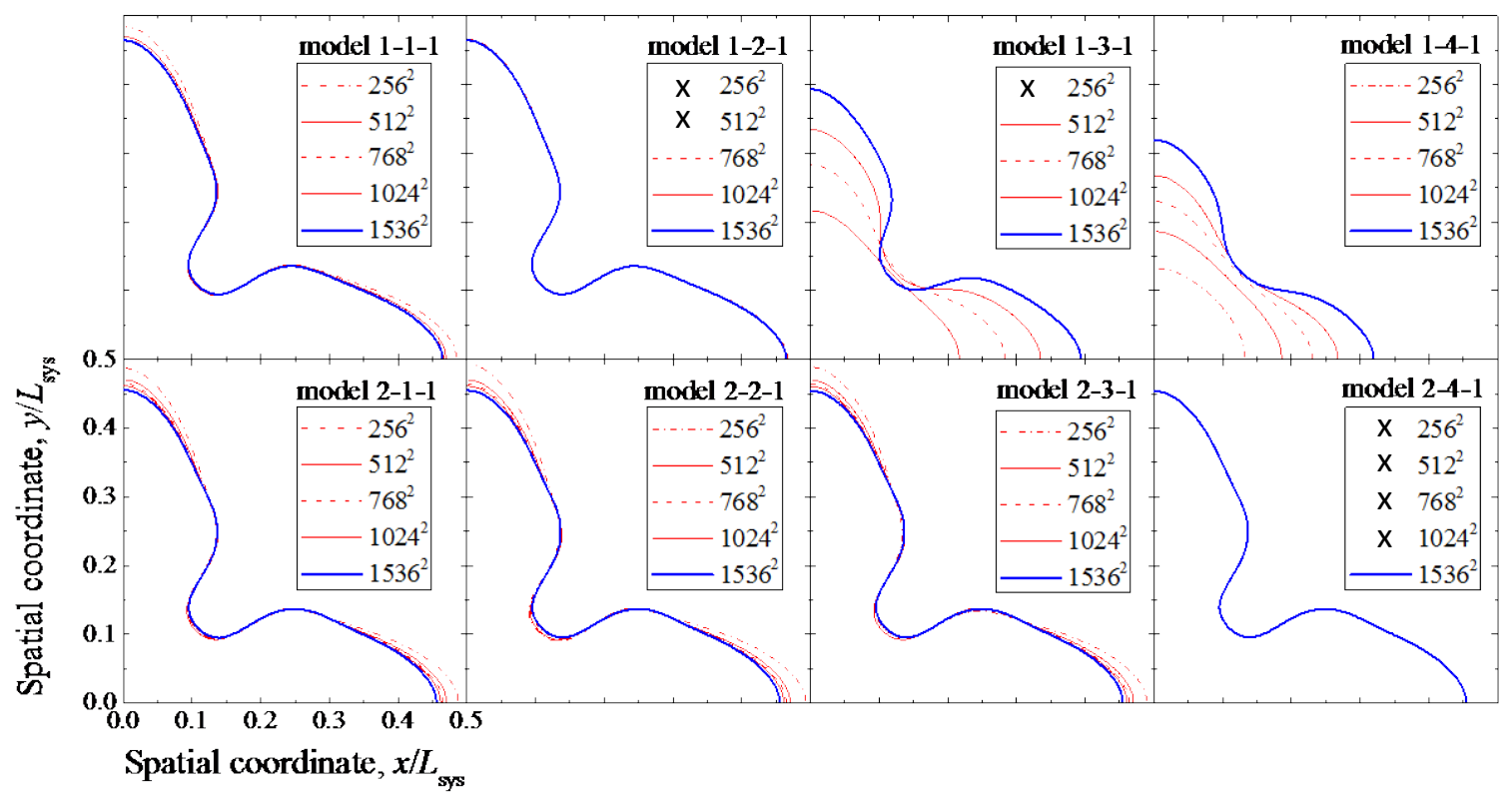

Fig. 5. Shapes of a dendrite during isothermal solidification calculated for $\Omega=0.2$ using eight different models. The name of the model is indicated in each figure and each line represents the $\phi=$ 0.0 contour line calculated using different values of $N_{p}$ as indicated in the legend. A cross mark in the legend indicates that the numerical simulation was unstable and the result was therefore not obtained for this value of $N_{p}$. 


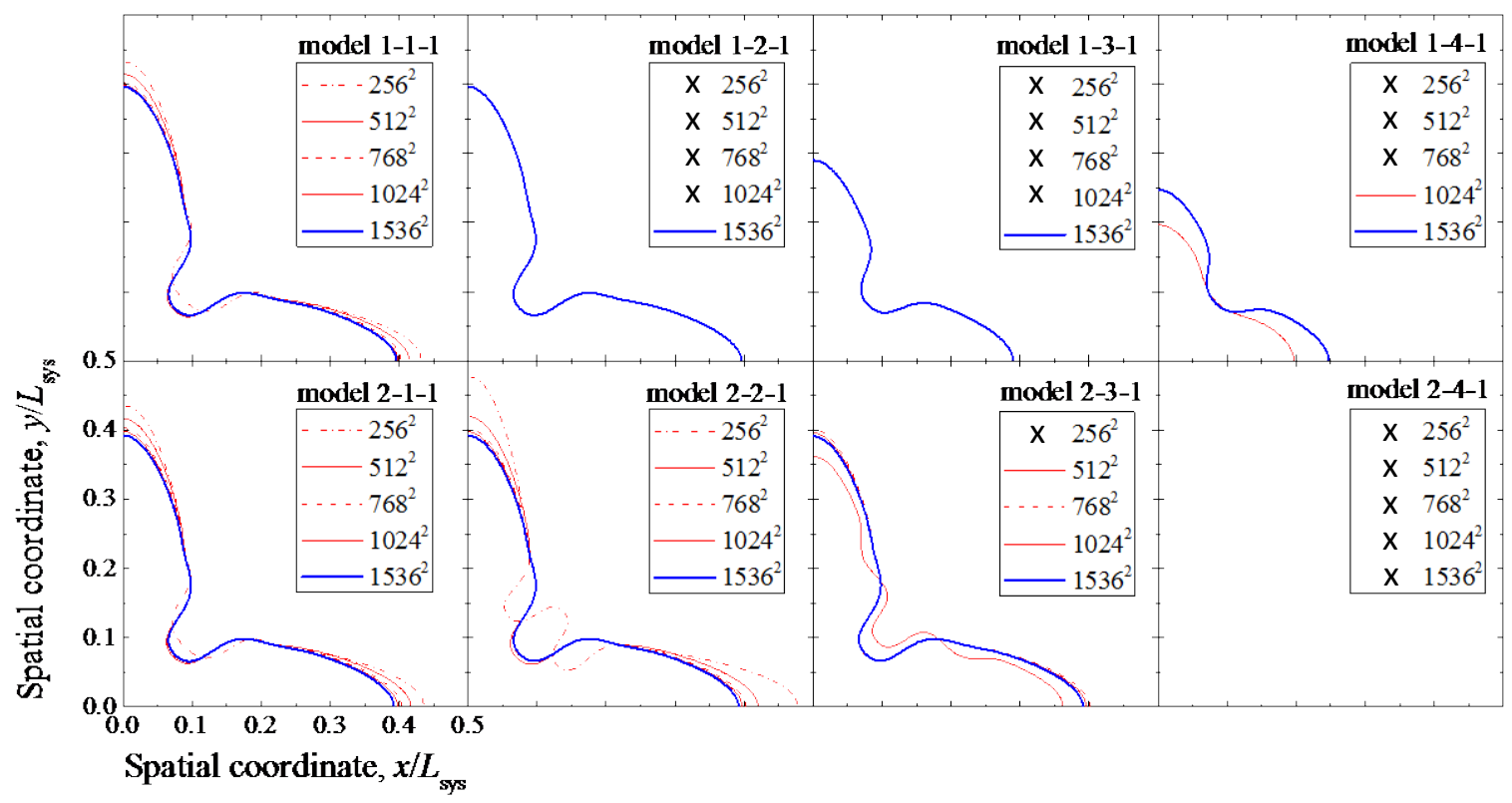

Fig. 6. Shapes of a dendrite during isothermal solidification calculated for $\Omega=0.3$ using eight different models. The name of the model is indicated in each figure and each line represents the $\phi=$ 0.0 contour lines calculated using different values of $N_{p}$ as indicated in the legend. A cross mark in the legend indicates that the numerical simulation was unstable and the result was therefore not obtained for this value of $N_{p}$. 
(a)

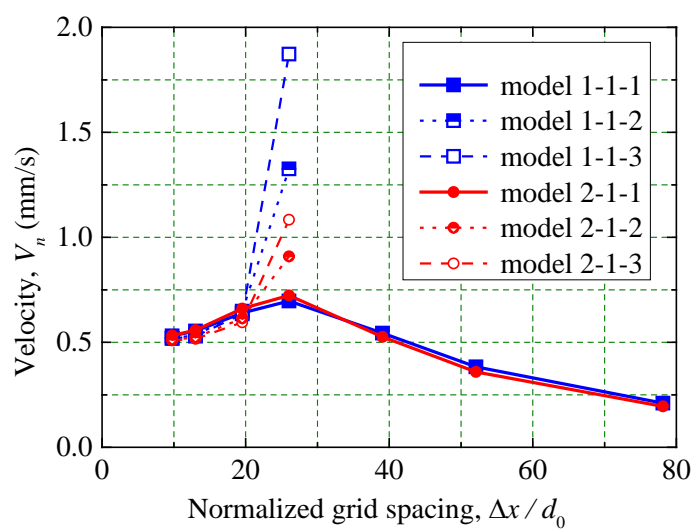

(c)

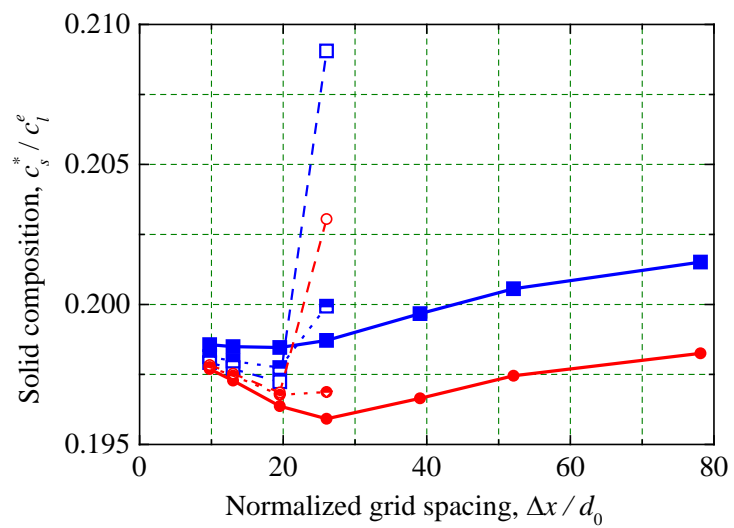

(b)

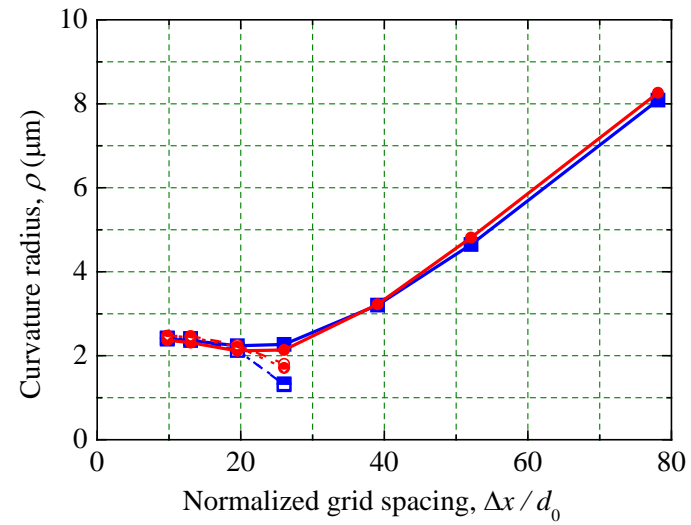

(d)

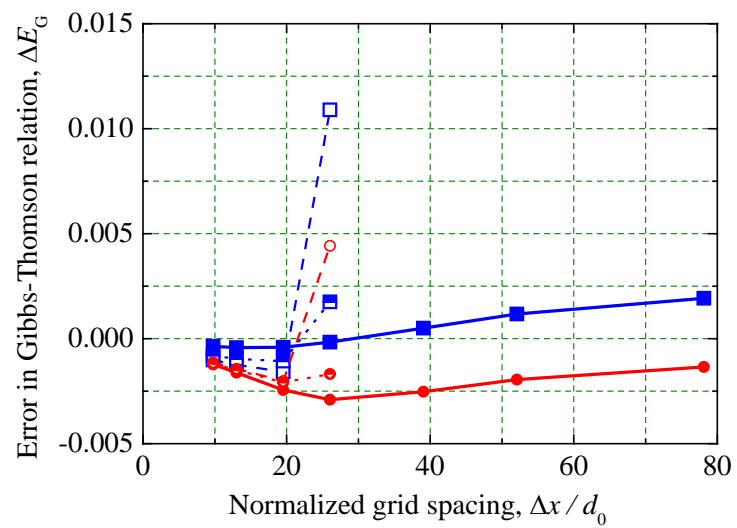

Fig. 7. Dependence of (a) velocity, (b) curvature radius, (c) solid composition and (d) error in Gibbs-Thomson relation on $\Delta x$ calculated for $\Omega=0.3, L_{\text {sys }}=4 \times 10^{-4} \mathrm{~m}$ and $n_{W}=1.25$. In each figure, the different symbols represent the results for the different models as specified in the legend in (a). 
(a)

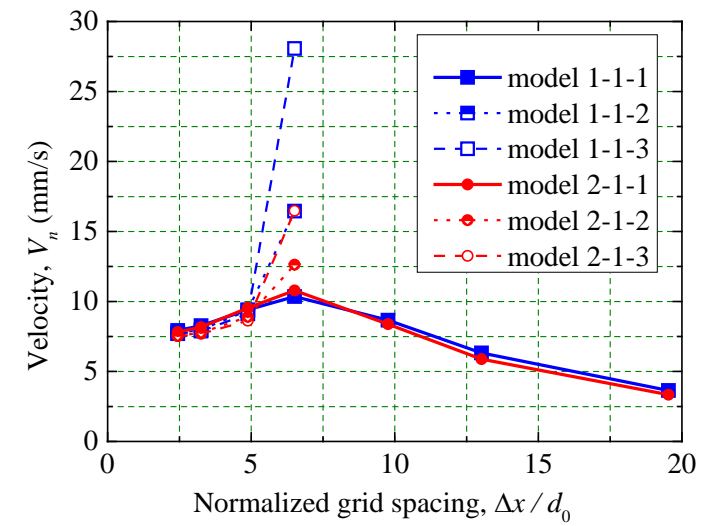

(c)

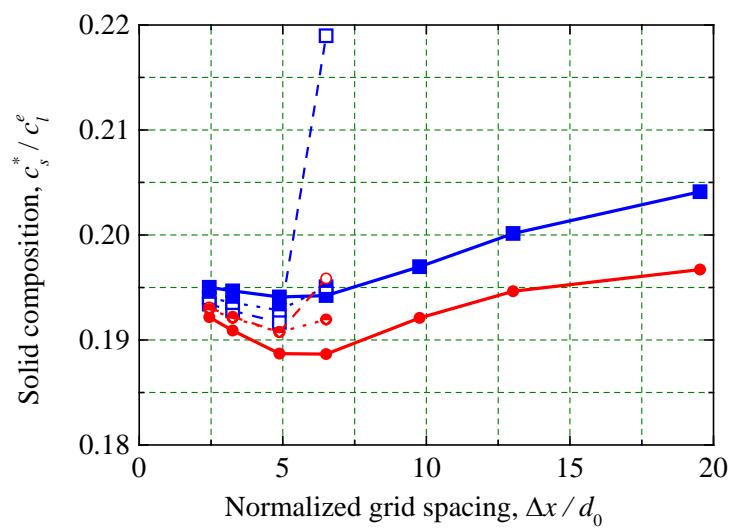

(b)

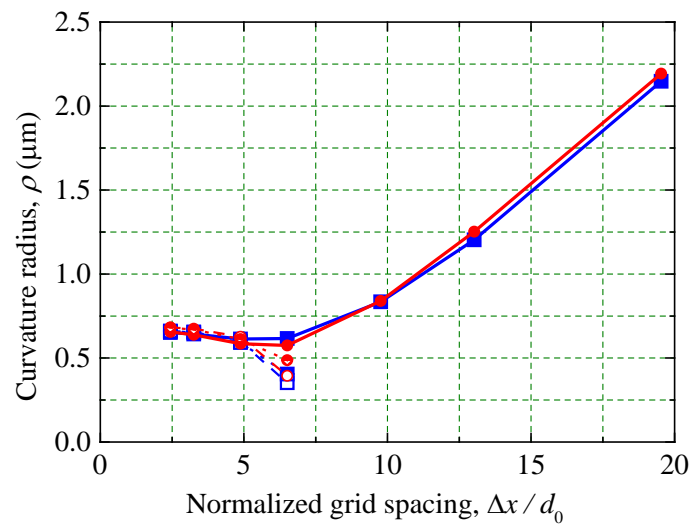

(d)

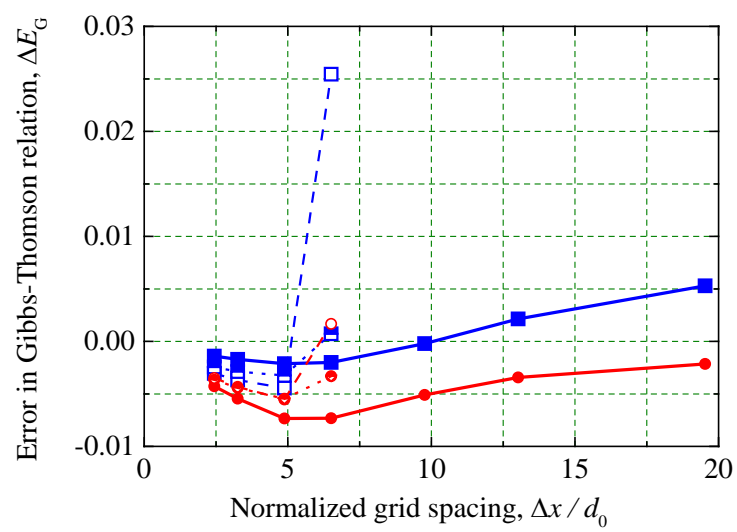

Fig. 8. Dependence of (a) velocity, (b) curvature radius, (c) solid composition and (d) error in Gibbs-Thomson relation on $\Delta x$ calculated for $\Omega=0.5, L_{\text {sys }}=1 \times 10^{-4} \mathrm{~m}$ and $n_{W}=1.25$. In each figure, the different symbols represent the results for the different models as specified in the legend in (a). 
(a)

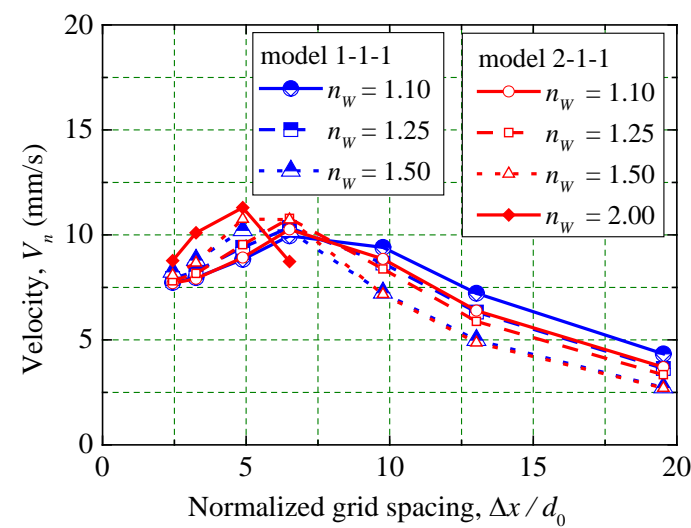

(c)

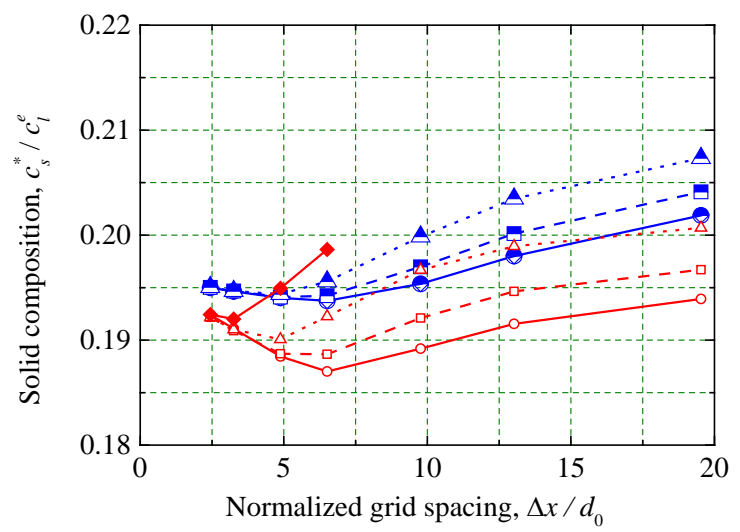

(b)

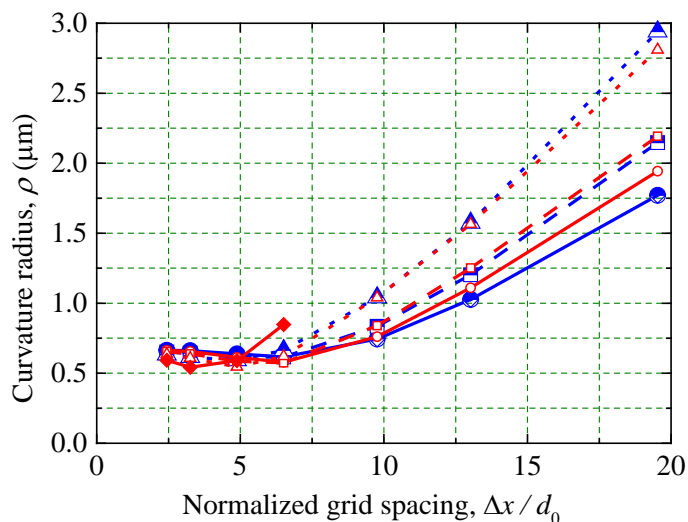

(d)

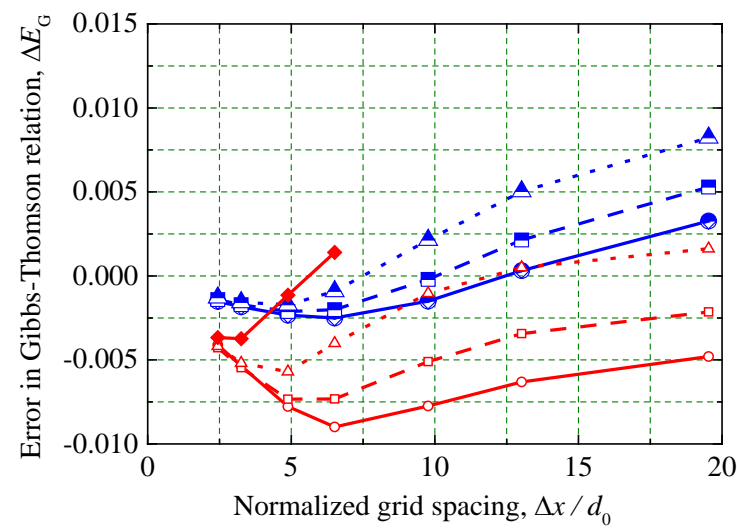

Fig. 9. Dependence of (a) velocity, (b) curvature radius, (c) solid composition and (d) error in Gibbs-Thomson relation on $\Delta x$ calculated for $\Omega=0.5$ and $L_{s y s}=1 \times 10^{-4} \mathrm{~m}$. In each figure, the different symbols represent the results for the different models with different values of $n_{W}$ specified in the legend in (a). 
(a)

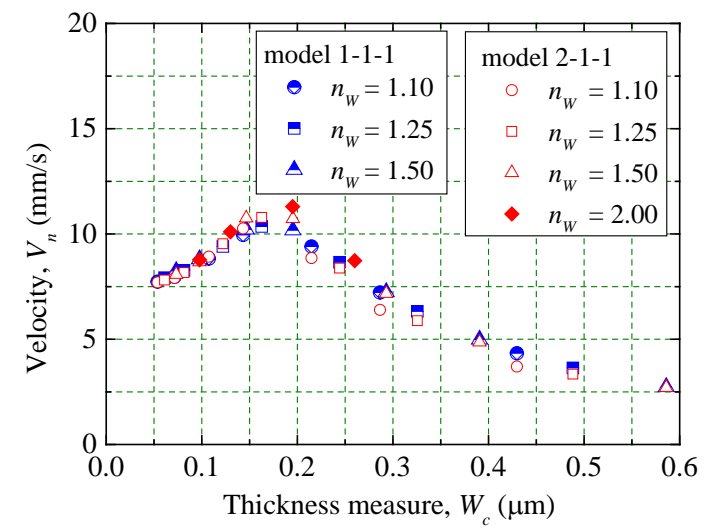

(c)

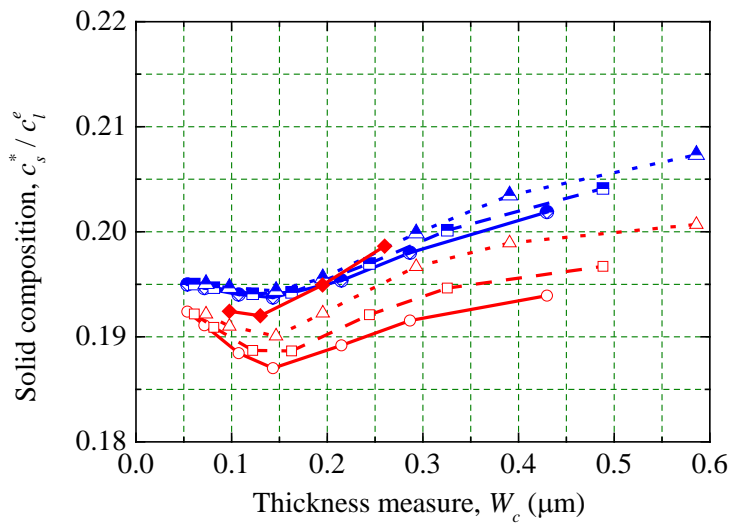

(b)

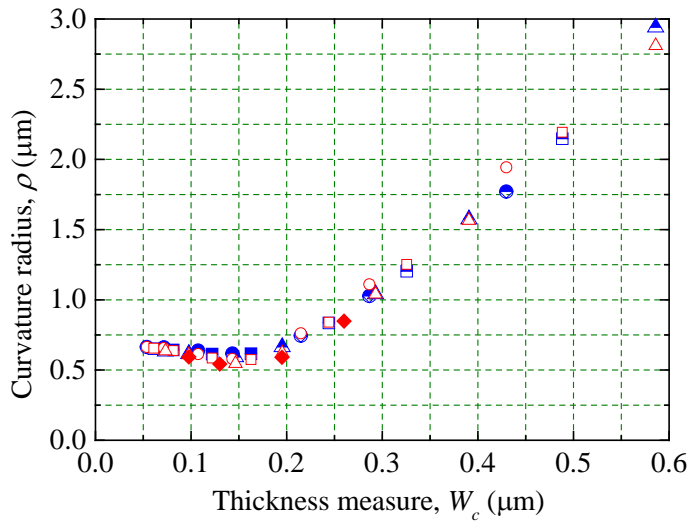

(d)

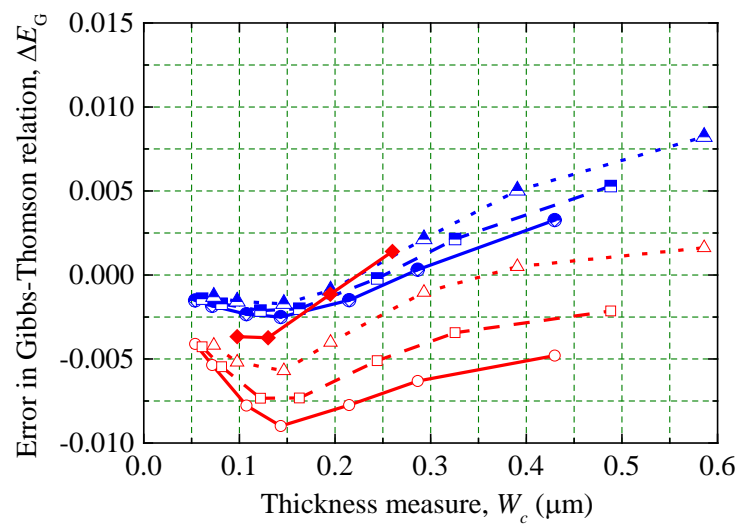

Fig. 10. Dependence of (a) velocity, (b) curvature radius, (c) solid composition and (d) error in Gibbs-Thomson relation on thickness measure calculated for $\Omega=0.5$ and $L_{\text {sys }}=1 \times 10^{-4} \mathrm{~m}$. In each figure, the different symbols represent the results for the different models with different values of $n_{W}$ specified in the legend in (a). 


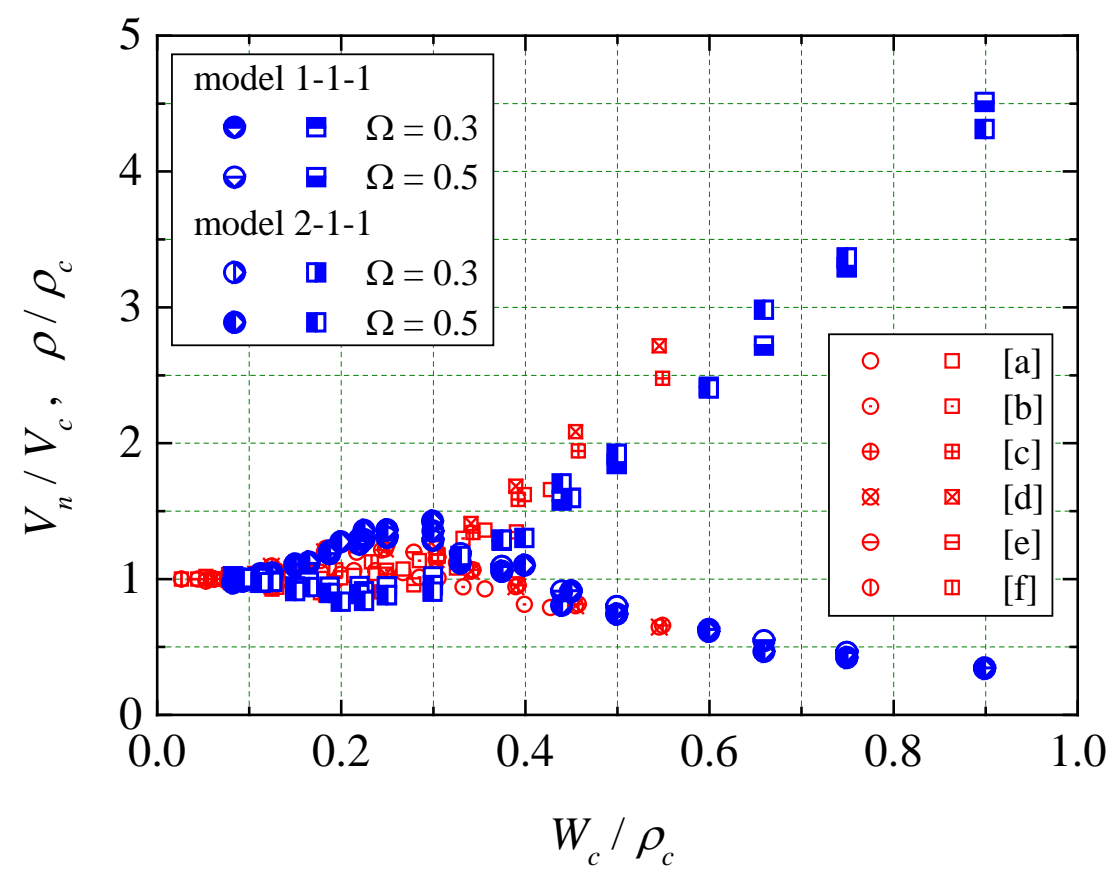

Fig. 11. Convergence behavior of $V_{n}$ (circles) and $\rho$ (squares) calculated with models 1-1-1 and 2-1-1 for $\Omega=0.3$ (Fig. 7) and $\Omega=0.5$ (Fig. 9). Data [a] and [b] are the results for isothermal solidification in binary alloys shown in Figs. 4 and 5 of Ref. [19], respectively. Data [c] and [d] are those for non-isothermal solidification shown in Figs. 2 and 3 of Ref. [21], respectively. Data [e] and [f] are the results for isothermal and non-isothermal solidification in a ternary alloy shown in Figs. 4 and 5 of Ref. [21], respectively. 